\title{
Highly Diastereoselective Synthesis of Homoallylic Alcohols Bearing Adjacent Quaternary Centers Using Substituted Allylic Zinc Reagents**
}

\author{
Hongjun Ren, Guillaume Dunet, Peter Mayer and Paul Knochel* \\ Department Chemie, Ludwig-Maximilians-Universität, Butenandtstrasse 5-13, 81377, \\ München (Germany).
}

\section{Paul.Knochel@cup.uni-muenchen.de}

\section{Supporting Information}

\section{General}

All reactions were carried out under nitrogen using standard Schlenk techniques. Melting points are uncorrected. ${ }^{1} \mathrm{H}$ and ${ }^{13} \mathrm{C}$ NMR spectra were recorded on a Bruker AMX 300 or AMX 600 or Varian VXR $400 \mathrm{~S}$ instrument. Chemical shifts are given as ppm relative to the residual solvent peak (chloroform- $d 1: 7.26 \mathrm{ppm} / 77.0 \mathrm{ppm}$; benzene- $d 67.16 \mathrm{ppm} / 128.0 \mathrm{ppm}$ ). IR spectra were recorded on a Perkin Elmer 1420 Infrared Spectrometer. Mass spectra were recorded on a Finnigan Mat 95 Q spectrometer. Column chromatography purification was performed on Merck silica gel 60 (230-400 mesh ASTM). THF and toluene were dried with sodium/benzophenone and distilled. Elemental analysis was performed on an Elementar vario EL and with a Metrohm Titroprocessor 686. Yields referred to isolated yields of compounds estimated to be $>95 \%$ pure as determined by ${ }^{1} \mathrm{H}-\mathrm{NMR}$, capillary GC and combustion analysis (new compounds).

\section{Synthesis of 3-chloro-1-methyl-cyclohexene}<smiles>CC1=CC(O)CCC1</smiles>

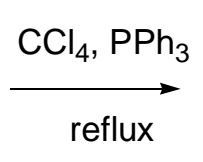

Triphenylphosphine $(14.70 \mathrm{~g}, 56.0 \mathrm{mmol})$ was slowly added to a solution of 3-methylcyclohex-2-enol $(4.50 \mathrm{~g}, 40.0 \mathrm{mmol})$ in carbon tetrachloride $(50 \mathrm{~mL})$ and the resulting mixture was refluxed for $5 \mathrm{~h}$. Then, the mixture was cooled and pentane $(150 \mathrm{~mL})$ was added. The suspension was filtered off and washed with pentane $(50 \mathrm{~mL})$. The solvent was removed under vacuum. Distillation $\left(40{ }^{\circ} \mathrm{C} / 3.0 \mathrm{mmHg}\right)$ of the residual oil provided the compound I $(3.13 \mathrm{~g}, 60 \%)$ as a colourless oil, as a ratio of 90: 10 mixture of I: II. The mixture was used in the subsequent step without further purification.

${ }^{1} \mathbf{H}$ NMR $\left(\mathrm{CDCl}_{3}, 300 \mathrm{MHz}\right):$ 5.50-5.60 (m, $\left.1 \mathrm{H}\right), 4.60-4.70(\mathrm{~m}, 1 \mathrm{H}), 1.80-2.10(\mathrm{~m}, 6 \mathrm{H})$, 1.68 (s, $3 \mathrm{H})$;

${ }^{13} \mathrm{C}$ NMR $\left(\mathrm{CDCl}_{3}, 75 \mathrm{MHz}\right): 139.8,122.6,57.1,32.0,29.8,23.7,18.5$;

IR (neat): 2934 (m), 1665 (m), $1444(\mathrm{~s}), 1436(\mathrm{w}), 1222(\mathrm{~s}) \mathrm{cm}^{-1}$;

MS (EI, $70 \mathrm{ev}): \mathrm{m} / \mathrm{z}(\%)=132\left(\mathrm{M}^{+}\left({ }^{37} \mathrm{Cl}\right), 9\right), 130$ (3), 95 (100), 79 (29), 67 (28).

The spectral date is in accordance with that reported in the literature. ${ }^{1}$

\section{Synthesis of 2-chloromethyl-6,6-dimethyl-bicyclo[3.1.1]hept-2-ene}

\footnotetext{
${ }^{1}$ T. Carrillo-Marquez, L. Caggiano, R. F. W. Jackson, U. Grabowska, A. Rae, M. J. Tozer, Org. Biomol. Chem., 2005, 3, 4117.
} 

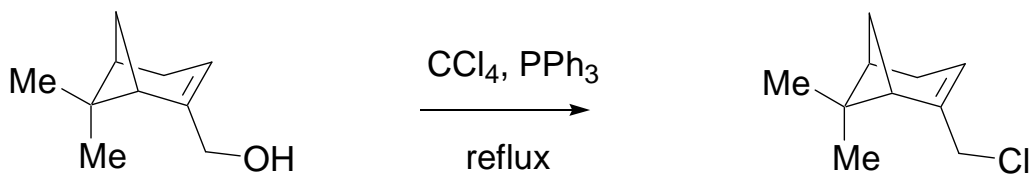

Triphenylphosphine $(36.68 \mathrm{~g}, 140 \mathrm{mmol})$ was slowly added to a solution of (-)-myrtenol $(15.20 \mathrm{~g}, 100 \mathrm{mmol})$ in carbon tetrachloride $(140 \mathrm{~mL})$ and the resulting mixture was refluxed for $5 \mathrm{~h}$. After the mixture was cooled, the pentane $(250 \mathrm{~mL})$ was added. The suspension was filtered off and washed with pentane $(100 \mathrm{~mL})$. The solvent was removed under vacuum. Distillation $\left(40{ }^{\circ} \mathrm{C} / 2.0 \mathrm{mmHg}\right)$ of the oil provided the compound $(12.44 \mathrm{~g}, 73 \%)$ as a colourless oil. $[\alpha]_{\mathrm{D}}^{20}=-40\left(\mathrm{c}=0.8, \mathrm{CH}_{2} \mathrm{Cl}_{2}\right)$.

${ }^{1} \mathrm{H} \mathrm{NMR}\left(\mathrm{CDCl}_{3}, 600 \mathrm{MHz}\right): 5.60(\mathrm{~s}, 1 \mathrm{H}), 3.94-4.01(\mathrm{~m}, 2 \mathrm{H}), 2.41\left(\mathrm{dt}, J_{1}=8.8 \mathrm{~Hz}, J_{2}=5.5\right.$ $\mathrm{Hz}, 1 \mathrm{H}), 2.20-2.32$ (m, $3 \mathrm{H}), 2.06-2.11(\mathrm{~m}, 1 \mathrm{H}), 1.29$ (s, $3 \mathrm{H}), 1.16$ (d, J = 8.8 Hz, $1 \mathrm{H}), 0.82$ $(\mathrm{s}, 3 \mathrm{H})$;

${ }^{13} \mathbf{C ~ N M R}\left(\mathrm{CDCl}_{3}, 150 \mathrm{MHz}\right): 144.1,122.4,48.6,44.2,40.4,38.0,31.5,31.2,26.0,21.1$;

IR (neat): 2919 (m), 1650 (w), 1469 (m), 1429 (m), 1366 (m), 1256 (s) cm $\mathrm{cm}^{-1}$;

MS (EI, $70 \mathrm{ev}): \mathrm{m} / \mathrm{z}(\%)=172\left(\mathrm{M}^{+}, 1\right), 170$ (3), 126 (13), 91 (100), 79 (15);

HRMS (EI): calcd. for $\mathrm{C}_{10} \mathrm{H}_{15} \mathrm{Cl}\left({ }^{35} \mathrm{Cl}\right)$ : 170.0862 ; found: $170.0849\left({ }^{35} \mathrm{Cl}\right)$.

\section{Preparation of 2-cyclohexenylzinc chloride (1a)}

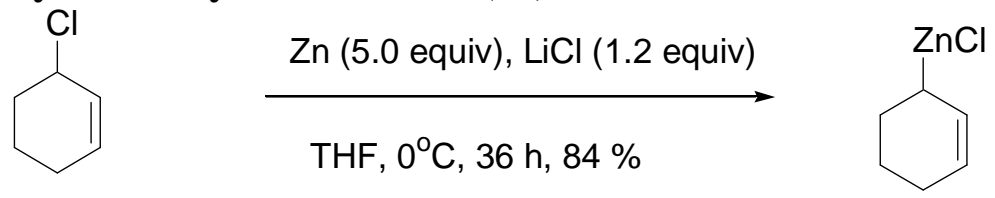

Zinc dust ${ }^{2}(3.20 \mathrm{~g}, 50.0 \mathrm{mmol})$ and dry $\mathrm{LiCl}(500 \mathrm{mg}, 12.0 \mathrm{mmol})$ were covered with dry THF ( $5 \mathrm{~mL})$ and activated by the addition of a few drops of 1,2-dibromoethane and TMSCl. After stirring for $10 \mathrm{~min}$, a solution of 3-chloro-cyclohexene (1.17 g, $10.0 \mathrm{mmol})$ in THF (10 $\mathrm{mL}$ ) was added in with a syringe pump at $0{ }^{\circ} \mathrm{C}$ within $2 \mathrm{~h}$. The resulting mixture was stirred under nitrogen at $0{ }^{\circ} \mathrm{C}$ for $36 \mathrm{~h}$. The zinc suspension was settled using centrifuge machine. The concentration and the yield were determined as follows:

Iodine $(254 \mathrm{mg}, 1.0 \mathrm{mmol})$ was placed into a dry $10 \mathrm{~mL}$ round-bottomed flask equipped with a magnetic stirrer bar and septum under nitrogen. Dry THF $(5 \mathrm{~mL})$ was added with syringe. The allylic zinc solution was added dropwise with syringe until the red colour disappeared. The volume of the allylic zinc reagent was determined and the following equation used to determine the molarity of the solution: molarity of allylic zinc reagent $=1 /$ volume of allylic zinc reagent.

The concentration of the allylic zinc reagent was $0.60 \mathrm{~mol} / \mathrm{l}$; volume $=14.0 \mathrm{~mL}$; yield: $84 \%$.

\section{Preparation of 2-cyclopentenylzinc chloride (1b)}

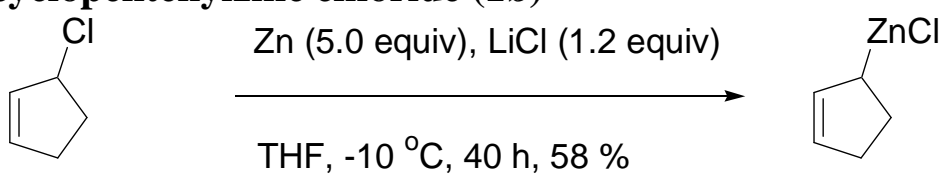

Zinc dust (3.20 g, $50.0 \mathrm{mmol})$ and dry $\mathrm{LiCl}(500 \mathrm{mg}, 12.0 \mathrm{mmol})$ were covered with dry THF $(5 \mathrm{~mL})$ and activated by the addition of a few drops of 1, 2-dibromoethane and TMSCl. After stirring for $10 \mathrm{~min}$, a solution of 3-chloro-cyclopentene $(1.03 \mathrm{~g}, 10.0 \mathrm{mmol})$ in THF $(10 \mathrm{~mL})$ was added with a syringe pump at $-10{ }^{\circ} \mathrm{C}$ within $2 \mathrm{~h}$. The resulting mixture was stirred under nitrogen at $-10{ }^{\circ} \mathrm{C}$ for $40 \mathrm{~h}$. The zinc suspension was allowed to settle using a centrifuge machine. The concentration and the yield were determined as the method described above. The concentration of the allylic zinc reagent was $0.40 \mathrm{~mol} / \mathrm{l}$; volume $=14.5 \mathrm{~mL}$; yield: $58 \%$.

${ }^{2}$ From Aldrich. 


\section{Preparation of 1-methyl-1-cyclohexenylzinc chloride (1c)}<smiles>CC1=CC(Cl)CCC1</smiles>

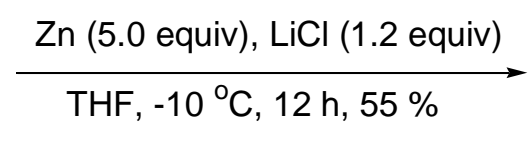<smiles>[CH]C1C=C(C)CCC1</smiles>

Zinc dust (3.20 g, $50.0 \mathrm{mmol})$ and dry $\mathrm{LiCl}(500 \mathrm{mg}, 12.0 \mathrm{mmol})$ were covered with dry THF $(5 \mathrm{~mL})$ and activated by the addition of a few drops of 1,2-dibromoethane and TMSCl After stirring for $10 \mathrm{~min}$, a solution of 3-chloro-1-methyl-cyclohexene (mixture of 3-chloro-1methyl-cyclohexene and 3-chloro-3-methyl-cyclohexene, $1.30 \mathrm{~g}, 10.0 \mathrm{mmol})$ in THF (10 mL) was added in with a syringe pump at $-10{ }^{\circ} \mathrm{C}$ within $2 \mathrm{~h}$. The resulting mixture was stirred under nitrogen at $-10{ }^{\circ} \mathrm{C}$ for $12 \mathrm{~h}$. The zinc suspension was allowed to settle using a centrifuge machine. The concentration and the yield were determined as the method described above. The concentration of allylic zinc reagent: $0.38 \mathrm{~mol} / \mathrm{l}$; volume $=14.5 \mathrm{~mL}$; yield: $55 \%$.

\section{Preparation of 2-methyl-6,6-dimethyl-bicyclo[3.1.1]hept-2-enylzinc chloride (1d)}
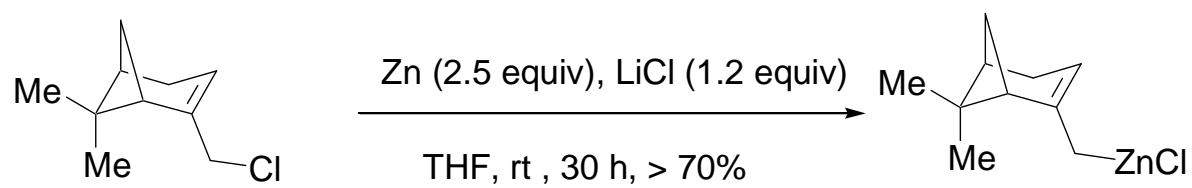

Zinc dust (1.60 g, $25.0 \mathrm{mmol})$ and dry $\mathrm{LiCl}(500 \mathrm{mg}, 12.0 \mathrm{mmol})$ were covered with dry THF $(5 \mathrm{~mL})$ and activated by the addition of a few drops of 1,2-dibromoethane and TMSCl. After stirring for $10 \mathrm{~min}$, a solution of 2-chloromethyl-6, 6-dimethyl-bicyclo[3.1.1]hept-2-ene (1.71 $\mathrm{g}, 10.0 \mathrm{mmol})$ in THF $(10 \mathrm{~mL})$ was added in with a syringe pump at room temperature within $2 \mathrm{~h}$. The resulting mixture was stirred under nitrogen at room temperature for $40 \mathrm{~h}$. The zinc suspension was allowed to settle using a centrifuge machine. The concentration and the yield were determined as the method described above.

Preparation of cinnamylzinc chloride (1e) :

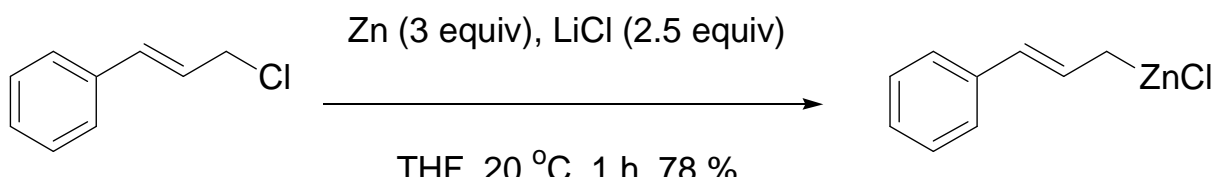

THF, $20{ }^{\circ} \mathrm{C}, 1 \mathrm{~h}, 78 \%$

Zinc dust $(2.00 \mathrm{~g}, 30.0 \mathrm{mmol})$ and dry $\mathrm{LiCl}(1.00 \mathrm{~g}, 24.0 \mathrm{mmol})$ were covered with dry THF $(5 \mathrm{~mL})$ and activated by the addition of a few drops of 1,2-dibromoethane and TMSCl. After stirring for $10 \mathrm{~min}$, a solution of cinnamyl chloride (1.52 g, $10.0 \mathrm{mmol})$ in THF (15 mL) was added at room temperature within $10 \mathrm{~min}$. The resulting mixture was stirred under nitrogen at room temperature for $1 \mathrm{~h}$. The zinc suspension was allowed to settle using a centrifuge machine. The concentration and the yield were determined as the method described above.

Preparation of cinnamylzinc chloride (1f) :

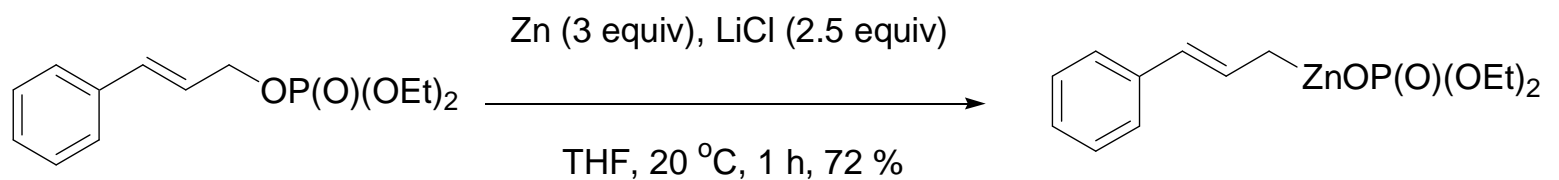

Zinc dust $(2.00 \mathrm{~g}, 30.0 \mathrm{mmol})$ and dry $\mathrm{LiCl}(1.00 \mathrm{~g}, 24.0 \mathrm{mmol})$ were covered with dry THF $(5 \mathrm{~mL})$ and activated by the addition of a few drops of 1,2-dibromoethane and TMSCl. After stirring for $10 \mathrm{~min}$, a solution of cinnamyl phosphate $(2.70 \mathrm{~g}, 10.0 \mathrm{mmol})$ in THF $(15 \mathrm{~mL})$ 
was added at room temperature within $10 \mathrm{~min}$. The resulting mixture was stirred under nitrogen at room temperature for $18 \mathrm{~h}$. The zinc suspension was allowed to settle using a centrifuge machine. The concentration and the yield were determined as the method described above.

Typical procedure for preparation of homoallylic alcohols by the addition of an allylic zinc reagent to an aldehyde or a ketone.

Procedure A: The allylic zinc chloride $(1.2 \mathrm{mmol})$ was added to the solution of aldehyde or ketone $(1.0 \mathrm{mmol})$ in THF $(2 \mathrm{~mL})$ at $-78{ }^{\circ} \mathrm{C}$ and the resulting mixture was stirred at this temperature for $1 \mathrm{~h}$. After quenching with water $(10 \mathrm{~mL})$, the reaction mixture was extracted with ether $(3 \times 30 \mathrm{~mL})$. The combined extracts were washed with brine, dried over $\mathrm{Na}_{2} \mathrm{SO}_{4}$ and concentrated in vacuo. Purification by flash chromatography (The silica gel was buffered with $1 \% \mathrm{Et}_{3} \mathrm{~N}$ in pentane) provided the pure compound.

Synthesis of 4-\{(1R*)-cyclohex-2-enyl $\}$ - $\left\{\left(1 S^{*}\right)\right.$-hydroxy-ethyl $\}$-benzonitrile (3a)<smiles>CO[C@](C)(c1ccc(C#N)cc1)[C@H]1C=CCCC1</smiles>

It was prepared from 4-acetylbenzonitrile $(145 \mathrm{mg}, 1.0 \mathrm{mmol})$ and 2-cyclohexenylzinc chloride (1a, $1.2 \mathrm{mmol}$ ) according to Procedure A. Purification by flash chromatography (eluent: pentane: ether $=2: 1)$ provided the pure compound 3a $(220 \mathrm{mg}, 97 \%)$ as a colourless oil. dr > 99:1.

${ }^{1} \mathbf{H}$ NMR $\left(\mathrm{CDCl}_{3}, 300 \mathrm{MHz}\right): 7.55(\mathrm{~d}, J=8.3 \mathrm{~Hz}, 2 \mathrm{H}), 7.49(\mathrm{~d}, J=8.3 \mathrm{~Hz}, 2 \mathrm{H}), 5.86-5.94$ $(\mathrm{m}, 1 \mathrm{H}), 5.72(\mathrm{~d}, J=10.5 \mathrm{~Hz}, 1 \mathrm{H}), 2.43-2.53(\mathrm{~m}, 1 \mathrm{H}), 2.08(\mathrm{~s}, 1 \mathrm{H}), 1.84-1.94(\mathrm{~m}, 2 \mathrm{H})$, 1.59-1.69 (m, $1 \mathrm{H}), 1.54$ (s, $3 \mathrm{H}), 1.05-1.44(\mathrm{~m}, 3 \mathrm{H})$;

${ }^{13} \mathbf{C ~ N M R}\left(\mathrm{CDCl}_{3}, 75 \mathrm{MHz}\right): 152.5,132.3,131.6,126.0,125.2,118.8,109.9,75.7,46.1,27.8$, 24.9, 24.1, 21.5;

IR (neat): $3481(\mathrm{~m}), 2931(\mathrm{~m}), 2227(\mathrm{~m}), 1606(\mathrm{~m}), 1372(\mathrm{~m}) \mathrm{cm}^{-1}$;

MS (EI, $70 \mathrm{ev}): \mathrm{m} / \mathrm{z}(\%)=227$ (0.5), $146(100), 130(8), 102(7)$;

HRMS (EI): calcd. for $\mathrm{C}_{15} \mathrm{H}_{18} \mathrm{NO}\left(\mathrm{M}^{+}+\mathrm{H}\right): 228.1388$; found: $228.1388\left(\mathrm{M}^{+}+\mathrm{H}\right)$.

Synthesis of $\left(1 S^{*}\right)$-\{(1R*)-cyclohex-2-enyl\}-2-methyl-phenyl-propan-1-ol (3b)<smiles>OC(c1ccccc1)([C@H]1C=CCCC1)C(F)(F)F</smiles>

It was prepared from 2-methyl-1-phenyl-propan-1-one (148 mg, $1.0 \mathrm{mmol})$ and 2cyclohexenylzinc chloride (1a, $1.2 \mathrm{mmol})$ according to Procedure A. This reaction was carried out at $-30{ }^{\circ} \mathrm{C}$ for $12 \mathrm{~h}$. Purification by flash chromatography (eluent: pentane: ether = 100: 1) provided the pure compound $\mathbf{3 b}(216 \mathrm{mg}, 94 \%)$ as a colourless oil. $\mathrm{dr}>99: 1$.

${ }^{1} \mathbf{H}$ NMR $\left(\mathrm{CDCl}_{3}, 300 \mathrm{MHz}\right)$ : 7.22-7.47 (m, $\left.5 \mathrm{H}\right)$, 5.86-6.00 (m, $\left.2 \mathrm{H}\right)$, 2.85-3.05 (m, $\left.1 \mathrm{H}\right)$, 2.27-2.42 (m, $1 \mathrm{H}), 1.83-2.03(\mathrm{~m}, 2 \mathrm{H}), 1.69-1.80(\mathrm{~m}, 1 \mathrm{H}), 1.63(\mathrm{~s}, 1 \mathrm{H}), 1.42-1.60(\mathrm{~m}, 2 \mathrm{H})$, $1.17-1.33(\mathrm{~m}, 1 \mathrm{H}), 0.91(\mathrm{~d}, J=7.0 \mathrm{~Hz}, 3 \mathrm{H}), 0.83(\mathrm{~d}, J=7.0 \mathrm{~Hz}, 3 \mathrm{H})$;

${ }^{13} \mathbf{C ~ N M R}\left(\mathrm{CDCl}_{3}, 75 \mathrm{MHz}\right): 142.2,131.3,127.2,126.6,126.4,126.1,80.4,41.6,34.7,25.2$, 24.4, 22.2, 17.6, 16.8;

IR (neat): $3568(\mathrm{w}), 2933(\mathrm{~m}), 1494(\mathrm{w}), 1468(\mathrm{~m}), 1445(\mathrm{~m}) \mathrm{cm}^{-1}$;

MS (EI, $70 \mathrm{ev}): \mathrm{m} / \mathrm{z}(\%)=213\left(\mathrm{M}^{+}-\mathrm{OH}, 1\right), 187$ (8), 149 (100), 105 (79);

HRMS (EI): calcd. for $\mathrm{C}_{16} \mathrm{H}_{20}\left(\mathrm{M}^{+}-\mathrm{H}_{2} \mathrm{O}\right): 212.1565$; found: $212.1574\left(\mathrm{M}^{+}-\mathrm{H}_{2} \mathrm{O}\right)$. 
To determine the structures of $\mathbf{3 a}$ and $\mathbf{3 b}$, another compound was synthesized and crystallized:

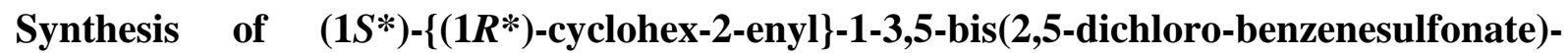
phenyl-ethanol (3I).
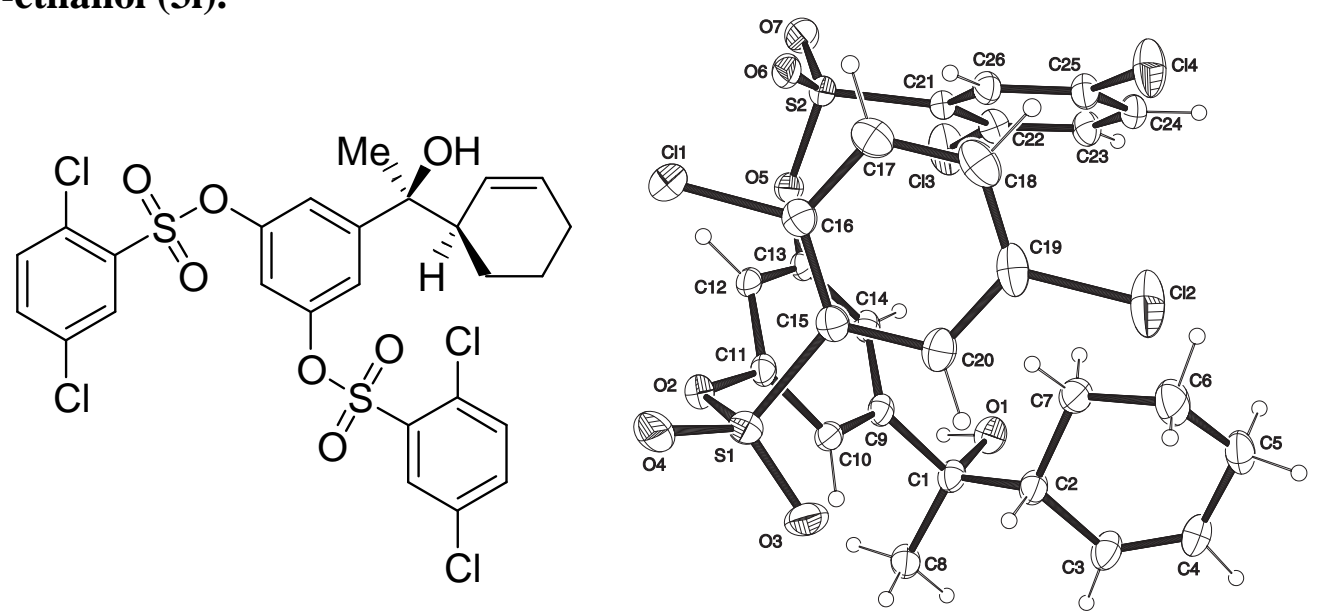

ORTEP representation of $\mathbf{3 1}$ with thermal ellipsoids set at $30 \%$ probability level (See the complete X-ray data at the end of the manuscript)

It was prepared from 3,5-bis(2,5-dichloro-benzenesulfonate)-acetophenone ${ }^{3}$ (570 mg, 1.0 mmol) and 2-cyclohexenylzinc chloride (1a, $1.2 \mathrm{mmol})$ according to Procedure $\mathbf{A}$. Purification by flash chromatography (eluent: pentane: ether $=6: 1$ ) provided the pure compound 3o (600 mg, 92\%) as a white solid, mp.: 149.6-150.8 ${ }^{\circ} \mathrm{C}$. dr > 99:1.

${ }^{1} \mathbf{H}$ NMR $\left(\mathrm{CDCl}_{3}, 300 \mathrm{MHz}\right): 7.78(\mathrm{~d}, J=2.6 \mathrm{~Hz}, 2 \mathrm{H}), 7.53(\mathrm{~s}, 4 \mathrm{H}), 7.03(\mathrm{~d}, J=2.6 \mathrm{~Hz}, 2$ $\mathrm{H}), 6.85(\mathrm{t}, J=2.6 \mathrm{~Hz}, 1 \mathrm{H}), 5.85-5.96(\mathrm{~m}, 1 \mathrm{H}), 5.59(\mathrm{~d}, J=10.6 \mathrm{~Hz}, 1 \mathrm{H}), 2.14-2.29(\mathrm{~m}, 1$ $\mathrm{H}), 1.50-2.00(\mathrm{~m}, 4 \mathrm{H}), 1.41(\mathrm{~s}, 3 \mathrm{H}), 1.19-1.36(\mathrm{~m}, 1 \mathrm{H}), 0.73-0.97(\mathrm{~m}, 2 \mathrm{H})$;

${ }^{13} \mathrm{C}$ NMR $\left(\mathrm{CDCl}_{3}, 75 \mathrm{MHz}\right): 151.2,149.0,135.2,134.1,133.4,133.3,133.0,132.1,131.5$, $124.7,118.3,114.5,75.3,46.2,27.9,25.0,24.0,21.7$;

IR (neat): 3559 (m), 2930 (s), 1612 (w), 1588 (s), 1452 (s), 1434, 1394, $1384 \mathrm{~cm}^{-1}$;

MS (EI, $70 \mathrm{ev}): \mathrm{m} / \mathrm{z}(\%)=634\left(\mathrm{M}^{+}-\mathrm{H} 2 \mathrm{O}, 0.5\right), 571$ (100), 360 (20), 145 (30);

Anal. Calcd for $\mathrm{C}_{26} \mathrm{H}_{22} \mathrm{C}_{14} \mathrm{O}_{7} \mathrm{~S}_{2}$ : C, 47.87; H, 3.40;

Found:

C, 47.76; H, 3.46.

Synthesis of $\left(1 S^{*}\right)$-(2-amino-5-chloro-phenyl)-\{(1R*)-cyclohex-2-enyl $\}$-methanol $(3 \mathrm{c})$.<smiles>Nc1ccc(Cl)cc1C(O)C1C=CCCC1</smiles>

It was prepared from 2-amino-5-chloro-benzaldehyde (156 mg, $1.0 \mathrm{mmol})$ and 2cyclohexenylzinc chloride (1a, $1.2 \mathrm{mmol})$ according to Procedure A. Purification by flash chromatography (eluent: pentane: ether $=1: 1)$ provided the pure compound $7(213 \mathrm{mg}, 90 \%)$ as a white solid, mp.: $112.0-112.5^{\circ} \mathrm{C}$. dr $>99: 1$ (determined by ${ }^{1} \mathrm{H}$ NMR).

${ }^{1} \mathbf{H}$ NMR $\left(\mathrm{CDCl}_{3}, 300 \mathrm{MHz}\right): 6.97-7.08(\mathrm{~m}, 2 \mathrm{H}), 6.55(\mathrm{~d}, J=8.8 \mathrm{~Hz}, 1 \mathrm{H}), 5.72-5.83(\mathrm{~m}, 1$ H), 5.17-5.29 (m, $1 \mathrm{H}), 4.43(\mathrm{~d}, J=7.9 \mathrm{~Hz}, 1 \mathrm{H}), 3.00-4.31(\mathrm{bs}, 2 \mathrm{H}), 2.61-2.74(\mathrm{~m}, 1 \mathrm{H})$, 1.94-2.05 (m, 2 H), 1.69-1.93 (m, 2 H), 1.45-1.65 (m, 2 H);

\footnotetext{
${ }^{3}$ Prepared from 3,5-dihydroxyacetophenone and 2,5-dichlorobenzenesulfonylchloride using a usual method.
} 
${ }^{13} \mathbf{C}$ NMR $\left(\mathrm{CDCl}_{3}, 75 \mathrm{MHz}\right): 143.2,130.4,128.2,128.00,127.97,127.5,122.8,117.9,76.7$, 39.6, 25.2, 24.9, 20.7;

IR (neat): $3384(\mathrm{~m}), 3357(\mathrm{~m}), 3162(\mathrm{~m}), 1487(\mathrm{~s}), 1420(\mathrm{~m}), 1200(\mathrm{~m}) \mathrm{cm}^{-1}$;

MS (EI, $70 \mathrm{ev}): \mathrm{m} / \mathrm{z}(\%)=219\left(\mathrm{M}^{+}-\mathrm{H}_{2} \mathrm{O}, 100\right), 191$ (91), 164 (48), 140 (79), 77 (29);

HRMS (EI): calcd. for $\mathrm{C}_{13} \mathrm{H}_{15} \mathrm{ClNO}\left(\mathrm{M}^{+}-\mathrm{H}\right)$ : 236.0842; found: $236.0852\left(\mathrm{M}^{+}-\mathrm{H}\right)$.

Synthesis of $\left(1 S^{*}\right)-\left\{\left(1 R^{*}\right)\right.$-cyclohex-2-enyl $\}-6-m e t h o x y-1,2,3,4-t e t r a h y d r o-n a p h t h a l e n-1-$ ol (3d)

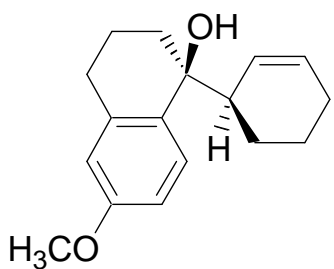

It was prepared from 6-methoxyl-1-tetralone $(176 \mathrm{mg}, 1.0 \mathrm{mmol})$ and 2-cyclohexenylzinc chloride (1a, $1.2 \mathrm{mmol}$ ) according to Procedure A. Purification by flash chromatography (eluent: pentane: ether $=3: 1)$ provided the pure compound $\mathbf{3 d}(250 \mathrm{mg}, 97 \%)$ as a colourless oil. dr $>$ 97:3.

${ }^{1} \mathbf{H}$ NMR $\left(\mathrm{CDCl}_{3}, 400 \mathrm{MHz}\right): 7.45(\mathrm{~d}, J=8.6 \mathrm{~Hz}, 1 \mathrm{H}), 6.76\left(\mathrm{dd}, J_{1}=8.6 \mathrm{~Hz}, J_{2}=2.7 \mathrm{~Hz}, 1\right.$ H), $6.59(\mathrm{~d}, J=2.7 \mathrm{~Hz}, 1 \mathrm{H}), 5.57-5.67(\mathrm{~m}, 1 \mathrm{H}), 4.97-5.06(\mathrm{~m}, 1 \mathrm{H}), 3.76(\mathrm{~s}, 3 \mathrm{H}), 2.84-2.93$ (m, $1 \mathrm{H}), 2.55-2.75(\mathrm{~m}, 2 \mathrm{H}), 1.65-2.08(\mathrm{~m}, 9 \mathrm{H}), 1.44-1.62(\mathrm{~m}, 2 \mathrm{H})$;

${ }^{13} \mathbf{C}$ NMR $\left(\mathrm{CDCl}_{3}, 100 \mathrm{MHz}\right): 158.2,139.7,133.4,128.9,128.4,127.8,113.0,112.7,73.7$, 55.0, 46.4, 33.1, 30.7, 25.3, 23.4, 22.3, 19.3;

IR (neat): 3446 (m), 2928 (s), 1607 (s), 1498 (s), $1253(\mathrm{~s}) \mathrm{cm}^{-1}$;

MS (EI, $70 \mathrm{ev}): \mathrm{m} / \mathrm{z}(\%)=240\left(\mathrm{M}^{+}-\mathrm{H}_{2} \mathrm{O}, 100\right), 225$ (9), 211 (22), 199 (33), 159 (24);

HRMS (EI): calcd. for $\mathrm{C}_{17} \mathrm{H}_{20} \mathrm{O}\left(\mathrm{M}^{+}-\mathrm{H}_{2} \mathrm{O}\right): 240,1514$; found: $240.1520\left(\mathrm{M}^{+}-\mathrm{H}_{2} \mathrm{O}\right)$.

Synthesis of $\left(1 S^{*}\right)-\left\{\left(1 R^{*}\right)\right.$-cyclohex-2-enyl $\}$-ferrocenyl-ethanol $(3 \mathrm{e})$.
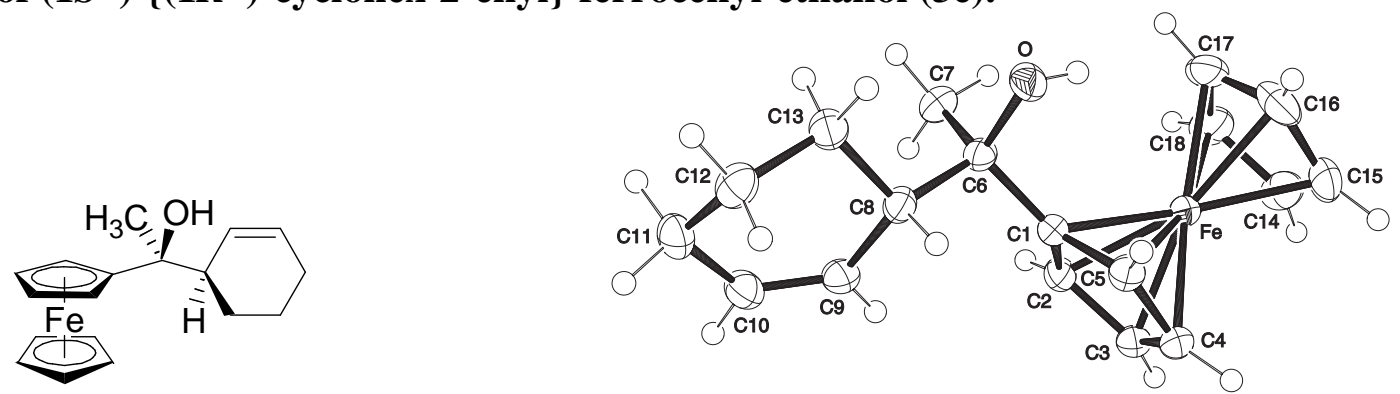

ORTEP representation of $\mathbf{3 e}$ with thermal ellipsoids set at $30 \%$ probability level (See the complete X-ray data at the end of the manuscript).

It was prepared from acetylferrocene $(228 \mathrm{mg}, 1.0 \mathrm{mmol})$ and 2-cyclohexenylzinc chloride (1a, $1.2 \mathrm{mmol}$ ) according to Procedure A. Purification by flash chromatography (eluent: pentane: ether $=10: 1)$ provided the pure compound 3e $(288 \mathrm{mg}, 93 \%)$ as a red solid, $\mathrm{mp}$. : 83.1-84. ${ }^{\circ} \mathrm{C} . \mathrm{dr}>99: 1$.

${ }^{1} \mathbf{H}$ NMR $\left(\mathrm{C}_{6} \mathrm{D}_{6}, 400 \mathrm{MHz}\right): 5.81-5.88(\mathrm{~m}, 1 \mathrm{H}), 5.67-5.75(\mathrm{~m}, 1 \mathrm{H}), 4.24-4.29(\mathrm{~m}, 1 \mathrm{H}), 3.99$ (s, $5 \mathrm{H}), 3.89-3.92(\mathrm{~m}, 2 \mathrm{H}), 3.86-3.88(\mathrm{~m}, 1 \mathrm{H}), 2.46-2.56(\mathrm{~m}, 1 \mathrm{H}), 2.09(\mathrm{~s}, 1 \mathrm{H}), 1.94-2.03$ $(\mathrm{m}, 1 \mathrm{H}), 1.76-1.85(\mathrm{~m}, 2 \mathrm{H}), 1.60-1.70(\mathrm{~m}, 1 \mathrm{H}), 1.49(\mathrm{~s}, 3 \mathrm{H}), 1.32-1.52(\mathrm{~m}, 2 \mathrm{H})$;

${ }^{13} \mathrm{C}$ NMR $\left(\mathrm{C}_{6} \mathrm{D}_{6}, 100 \mathrm{MHz}\right): 128.7,128.6,68.6,68.5,68.1,67.6,66.1,47.8,25.9,25.5,25.2$, 22.6;

IR (neat): 3545 (m), 2926 (m), $1446(\mathrm{~m}), 1369(\mathrm{~m}), 1318(\mathrm{~s}) \mathrm{cm}^{-1}$;

MS (EI, $70 \mathrm{ev}): \mathrm{m} / \mathrm{z}(\%)=292\left(\mathrm{M}^{+}-\mathrm{H}_{2} \mathrm{O}, 100\right), 275$ (10), 225 (11), 166 (9), 121 (12);

HRMS (EI): calcd. for $\mathrm{C}_{18} \mathrm{H}_{20} \mathrm{Fe}\left(\mathrm{M}^{+}-\mathrm{H}_{2} \mathrm{O}\right)$ : 292.0914; found: $292.0906\left(\mathrm{M}^{+}-\mathrm{H}_{2} \mathrm{O}\right)$. 
Synthesis of $\left(1 S^{*}\right)$-2-chloro- $\left\{\left(1 R^{*}\right)\right.$-cyclohex-2-enyl $\}$-1-phenyl-ethanol (3f)<smiles>OC(CCl)(c1ccccc1)[C@H]1C=CCCC1</smiles>

It was prepared from 2-chloro-1-phenyl-ethanone (161 mg, $1.0 \mathrm{mmol})$ and 2cyclohexenylzinc chloride (1a, $1.2 \mathrm{mmol})$ according to Procedure A. Purification by flash chromatography (eluent: pentane: ether $=5: 1)$ provided the pure compound $4 \mathbf{a}(230 \mathrm{mg}$, 97\%) as a colourless oil. dr $>99: 1$.

${ }^{1} \mathbf{H}$ NMR $\left(\mathrm{CDCl}_{3}, 300 \mathrm{MHz}\right):$ 7.28-7.51 (m, $\left.5 \mathrm{H}\right)$, 5.85-5.93 (m, $\left.1 \mathrm{H}\right)$, 5.70-5.85 (m, $\left.1 \mathrm{H}\right)$, 3.99-4.20 (m, $2 \mathrm{H}), 2.70-2.82(\mathrm{~m}, 1 \mathrm{H}), 2.58(\mathrm{~s}, 1 \mathrm{H}), 1.84-2.10(\mathrm{~m}, 2 \mathrm{H}), 1.69-1.80(\mathrm{~m}, 1 \mathrm{H})$, $1.29-1.66(\mathrm{~m}, 3 \mathrm{H})$;

${ }^{13} \mathrm{C}$ NMR $\left(\mathrm{CDCl}_{3}, 75 \mathrm{MHz}\right): 141.9,131.1,127.9,127.1,126.0,125.4,77.9,52.4,44.3,24.9$, 24.3, 21.8;

IR (neat): 3550 (m), 1495 (m), 1446 (s), $1433(\mathrm{~m}), 1054(\mathrm{~m}) \mathrm{cm}^{-1}$;

MS (EI, $70 \mathrm{ev}): \mathrm{m} / \mathrm{z}(\%)=219\left(\mathrm{M}^{+}-\mathrm{H}_{2} \mathrm{O}, 0.1\right), 155$ (100), 105 (8), 91 (9), 77 (32);

HRMS (EI): calcd. for $\mathrm{C}_{14} \mathrm{H}_{16} \mathrm{Cl}\left(\mathrm{M}^{+}-\mathrm{OH}\right)$ : 219.0941; found: $219.0924\left(\mathrm{M}^{+}-\mathrm{OH}\right)$.

Synthesis of $\left(1 S^{*}\right)$-2-azido-\{(1R*)-cyclohex-2-enyl $\}-1-p h e n y l-e t h a n o l ~(3 g)$<smiles>N#CC(O)(c1ccccc1)[C@H]1C=CCCC1</smiles>

It was prepared from 2-azido-1-phenyl-ethanone $(161 \mathrm{mg}, 1.0 \mathrm{mmol})$ and 2-cyclohexenylzinc chloride (1a, $1.2 \mathrm{mmol}$ ) according to Procedure A. Purification by flash chromatography (eluent: pentane: ether $=5: 1)$ provided the pure compound $\mathbf{4 b}(226 \mathrm{mg}, 93 \%)$ as a colourless oil. dr > 99:1.

${ }^{1}$ H NMR $\left(\mathrm{CDCl}_{3}, 300 \mathrm{MHz}\right):$ 7.20-7.50 (m, $\left.5 \mathrm{H}\right)$, 5.84-5.96 (m, $\left.1 \mathrm{H}\right)$, 5.70-5.80 (m, $\left.1 \mathrm{H}\right)$, 3.74 (s, 2 H), 2.57-2.71 (m, $1 \mathrm{H}), 2.33$ (bs, $1 \mathrm{H}), 1.83-2.00$ (m, $2 \mathrm{H}), 1.62-1.75$ (m, $1 \mathrm{H}), 1.34-$ 1.49 (m, $2 \mathrm{H}), 1.17-1.31(\mathrm{~m}, 1 \mathrm{H})$;

${ }^{13} \mathbf{C ~ N M R}\left(\mathrm{CDCl}_{3}, 75 \mathrm{MHz}\right): 142.4,132.1,128.1,127.1,125.6,125.2,78.3,59.2,43.7,24.9$, 24.0, 21.6;

IR (neat): 3547 (w), 2130 (vs), 1495 (w), $1446(\mathrm{~m}) \mathrm{cm}^{-1}$;

MS (EI, $70 \mathrm{ev}): \mathrm{m} / \mathrm{z}(\%)=197\left(\mathrm{M}^{+}-\mathrm{H}_{2} \mathrm{O}-\mathrm{N}_{2}, 95\right), 169$ (100), 115 (8);

HRMS (ESI):calcd. for $\mathrm{C}_{15} \mathrm{H}_{18} \mathrm{~N}_{3} \mathrm{O}_{3}$ ([M+FA-H] $\left.]^{-}\right)$: 288.1348 ; found: 288.1375 ([M+FA-H $\left.]^{-}\right)$; calcd. for $\mathrm{C}_{16} \mathrm{H}_{20} \mathrm{~N}_{3} \mathrm{O}_{3}\left([\mathrm{M}+\mathrm{AA}-\mathrm{H}]^{-}\right)$: 302.1505 ; found: 302.1535 ([M+AA-H] $]^{-}$).

Synthesis of $\left(1 S^{*}\right)-(4-b r o m o-p h e n y l)-\left\{\left(1 R^{*}\right)\right.$-cyclohex-2-enyl $\}-2-(4-p h e n y l-[1,2,3]$ triazol1-yl)-ethanol (3g')
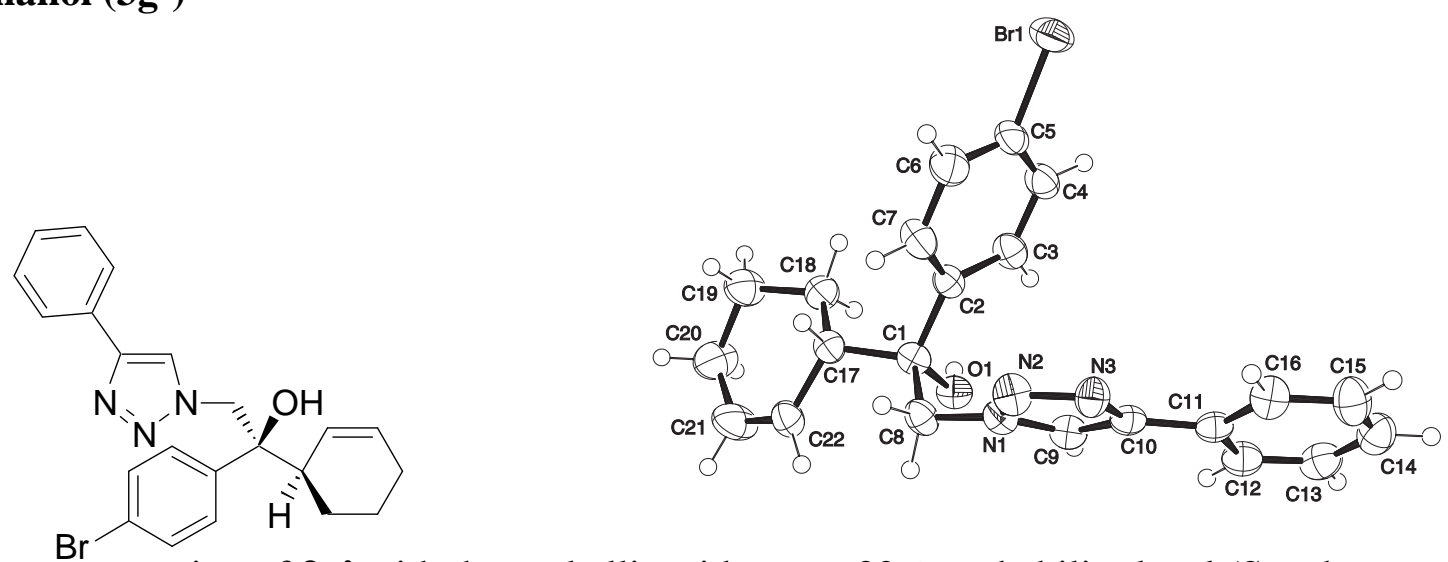

ORTEP representation of $\mathbf{3 g}$ ' with thermal ellipsoids set at $30 \%$ probability level (See the complete X-ray data at the end of the manuscript). 
2-Cyclohexenylzinc chloride 1a $(2.0 \mathrm{~mL}, 1.2 \mathrm{mmol}, 0.6 \mathrm{M}$ in THF) was added to the solution of 2-azido-1-(4-bromo-phenyl)ethanone $(240 \mathrm{mg}, 1.0 \mathrm{mmol})$ and ethynylbenzene $(153 \mathrm{mg}$, $1.5 \mathrm{mmol})$ in THF $(2.0 \mathrm{~mL})$ at $-78{ }^{\circ} \mathrm{C}$ and the resulting mixture was stirred for $1 \mathrm{~h}$ at this temperature. The solution of $\mathrm{CuCN} \cdot 2 \mathrm{LiCl}(0.05 \mathrm{~mL}, 5 \mathrm{~mol} \%, 1.0 \mathrm{M}$ in THF) was added and the reaction mixture was stirred overnight at room temperature. After quenching with water $(10 \mathrm{~mL})$, the reaction mixture was extracted with $\mathrm{CH}_{2} \mathrm{Cl}_{2}(3 \times 30 \mathrm{~mL})$. The combined extracts were washed with brine and dried over $\mathrm{Na}_{2} \mathrm{SO}_{4}$ and concentrated in vacuo. Purification by flash chromatography (eluent: pentane: ether $=10: 1$ then ether: $\mathrm{CH}_{2} \mathrm{Cl}_{2}=1: 1$ ) provided the pure compound 3g' (400 mg, 94\%) as a white solid, mp.: $157.0-158.5{ }^{\circ} \mathrm{C}$.

${ }^{1} \mathbf{H}$ NMR $\left(\mathrm{CDCl}_{3}, 600 \mathrm{MHz}\right): 7.66(\mathrm{~d}, J=7.6 \mathrm{~Hz}, 2 \mathrm{H}), 7.51(\mathrm{~s}, 1 \mathrm{H}), 7.32-7.41(\mathrm{~m}, 4 \mathrm{H}), 7.28$ (t, $J=7.5 \mathrm{~Hz}, 1 \mathrm{H}), 7.19(\mathrm{~d}, J=7.6 \mathrm{~Hz}, 2 \mathrm{H}), 5.99-6.05(\mathrm{~m}, 1 \mathrm{H}), 5.81(\mathrm{~d}, J=10.1 \mathrm{~Hz}, 1 \mathrm{H})$, $4.84(\mathrm{~d}, J=14.1 \mathrm{~Hz}, 1 \mathrm{H}), 4.77(\mathrm{~d}, J=14.1 \mathrm{~Hz}, 1 \mathrm{H}), 3.19$ (s, $1 \mathrm{H}), 2.68-2.76$ (m, $1 \mathrm{H}), 1.87-$ 2.02 (m, $2 \mathrm{H}), 1.64-1.75(\mathrm{~m}, 1 \mathrm{H}), 1.36-1.48(\mathrm{~m}, 2 \mathrm{H}), 1.18-1.30(\mathrm{~m}, 1 \mathrm{H})$, ;

${ }^{13} \mathrm{C}$ NMR $\left(\mathrm{CDCl}_{3}, 150 \mathrm{MHz}\right): 147.2,140.2,133.8,131.3,130.3,128.8,128.1,127.5,125.6$, $124.2,121.4,121.0,77.9,58.2,43.8,25.0,24.1,21.4$;

IR (neat): 3327 (m), 2939 (m), 1609 (m), $1590(\mathrm{~m}), 1487(\mathrm{~m}), 1466(\mathrm{~m}), 1005$ (s) cm ${ }^{-1}$;

MS (EI, $70 \mathrm{ev}): \mathrm{m} / \mathrm{z}(\%)=425\left(\mathrm{M}^{+}\left({ }^{81} \mathrm{Br}\right), 1\right), 423\left(\mathrm{M}^{+}\left({ }^{79} \mathrm{Br}\right), 1\right), 342(18), 296(6), 183(88)$, 159 (100), 130 (42);

HRMS (EI): calcd. for $\mathrm{C}_{22} \mathrm{H}_{22} \mathrm{BrN}_{3} \mathrm{O}\left(\mathrm{M}^{+},{ }^{79} \mathrm{Br}\right)$ : 423.0946 ; found: $423.0926\left(\mathrm{M}^{+},{ }^{79} \mathrm{Br}\right)$.

Synthesis of 4-\{(1R*)-cyclopent-2-enyl $\}$ - $\left\{\left(1 S^{*}\right)\right.$-hydroxy-ethyl $\}$-benzoic acid methyl ester (3h)<smiles>COC(=O)c1ccc([C@](C)(O)[C@H]2C=CCC2)cc1</smiles>

It was prepared from 4-acetyl-benzoic acid methyl ester (178 $\mathrm{mg}, 1.0 \mathrm{mmol}$ ) and 2cyclopentylzinc chloride (1b, $1.2 \mathrm{mmol})$ according to Procedure A. This reaction was carried out in THF $(5.0 \mathrm{~mL})$. Purification by flash chromatography (eluent: pentane: ether $=3: 1$ ) provided the pure compound $3 \mathbf{i}$ (236 $\mathrm{mg}, 96 \%)$ as a colourless oil. $\mathrm{dr}>99: 1$.

${ }^{1} \mathbf{H}$ NMR $\left(\mathrm{CDCl}_{3}, 300 \mathrm{MHz}\right): 7.96(\mathrm{~d}, J=8.5 \mathrm{~Hz}, 2 \mathrm{H}), 7.49(\mathrm{~d}, J=8.5 \mathrm{~Hz}, 2 \mathrm{H}), 5.95-6.00$ (m, $1 \mathrm{H}), 5.72-5.77(\mathrm{~m}, 1 \mathrm{H}), 3.87(\mathrm{~s}, 3 \mathrm{H}), 3.17-3.25(\mathrm{~m}, 1 \mathrm{H}), 2.10-2.33(\mathrm{~m}, 2 \mathrm{H}), 1.87$ (s, 1 $\mathrm{H}), 1.46-1.65(\mathrm{~m}, 2 \mathrm{H}), 1.53(\mathrm{~s}, 3 \mathrm{H})$;

${ }^{13} \mathbf{C}$ NMR $\left(\mathrm{CDCl}_{3}, 75 \mathrm{MHz}\right): 167.0,152.7,135.9,129.3$ (2 X C), 128.1, 125.2, 75.8, 57.1, 51.9, 32.2, 29.0, 24.6;

IR (neat): 3499 (m), 2951 (m), 1705 (s), 1609 (m), 1436 (m), 1406 (m), 1276 (vs) cm ${ }^{-1}$;

MS (EI, $70 \mathrm{ev}): \mathrm{m} / \mathrm{z}(\%)=228\left(\mathrm{M}^{+}-\mathrm{H}_{2} \mathrm{O}, 1\right), 179$ (100), 163 (8), 137 (9), 77 (9);

HRMS (EI): calcd. for $\mathrm{C}_{15} \mathrm{H}_{19} \mathrm{O}_{3}\left(\mathrm{M}^{+}+\mathrm{H}\right)$ : 247.1334; found: $247.1349\left(\mathrm{M}^{+}+\mathrm{H}\right)$.

Synthesis of $\left(1 S^{*}\right)-\left\{\left(1 R^{*}\right)\right.$-1-methyl-cyclohex-2-enyl $\}$-phenyl-methanol (3i)<smiles>C[C@@](O)(c1ccccc1)[C@H]1C=CCCC1</smiles>

It was prepared from benzaldehyde $(106 \mathrm{mg}, 1.0 \mathrm{mmol})$ and 1-methyl-1-cyclohexenylzinc chloride (1c, $1.2 \mathrm{mmol}$ ) according to Procedure A. Purification by flash chromatography (eluent: pentane: ether $=4: 1)$ provided the pure compound $\mathbf{3 i}(198 \mathrm{mg}, 98 \%)$ as a colourless oil. dr $>97: 3$.

${ }^{\mathbf{1}} \mathbf{H}$ NMR $\left(\mathrm{CDCl}_{3}, 300 \mathrm{MHz}\right): 7.25-7.36(\mathrm{~m}, 5 \mathrm{H}), 5.86\left(\mathrm{dt}, J_{1}=10.2 \mathrm{~Hz}, J_{2}=3.7 \mathrm{~Hz}, 1 \mathrm{H}\right)$, $5.49(\mathrm{~d}, J=10.2 \mathrm{~Hz}, 1 \mathrm{H}), 4.48(\mathrm{~d}, J=2.2 \mathrm{~Hz}, 1 \mathrm{H}), 1.99(\mathrm{~d}, J=2.2 \mathrm{~Hz}, 1 \mathrm{H}), 1.91-1.98(\mathrm{~m}, 2$ H), 1.81-1.91 (m, $1 \mathrm{H}), 1.47-1.76(\mathrm{~m}, 2 \mathrm{H}), 1.17-1.27(\mathrm{~m}, 1 \mathrm{H}), 0.93(\mathrm{~s}, 3 \mathrm{H})$; 
${ }^{13} \mathbf{C}$ NMR $\left(\mathrm{CDCl}_{3}, 75 \mathrm{MHz}\right): 140.6,133.7,129.4,127.9,127.4,127.2,80.8,40.4,29.7,25.1$, 23.7, 18.8;

IR (neat): 3439 (w), 2930 (m), 1493 (w), $1452(\mathrm{~m}), 1186(\mathrm{~m}), 1022(\mathrm{~s}) \mathrm{cm}^{-1}$;

MS (EI, $70 \mathrm{ev}): \mathrm{m} / \mathrm{z}(\%)=184\left(\mathrm{M}^{+}-\mathrm{H}_{2} \mathrm{O}, 0.5\right), 107$ (100), 96 (85), 79 (59), 67 (13);

HRMS (EI): calcd. for $\mathrm{C}_{14} \mathrm{H}_{17}\left(\mathrm{M}^{+}-\mathrm{OH}\right)$ : 185.1330; found: $185.1345\left(\mathrm{M}^{+}-\mathrm{OH}\right)$.

Synthesis of (1S*)-(4-bromo-phenyl)-1-\{(1R*)-1-methyl-cyclohex-2-enyl $\}$-ethanol $(3 \mathrm{j})$<smiles>C[C@](O)(c1ccc(Br)cc1)[C@H]1C=CCCC1</smiles>

It was prepared from 4-bromoacetophenone (199 $\mathrm{mg}, 1.0 \mathrm{mmol})$ and 1-methyl-1cyclohexenylzinc chloride (1c, $1.2 \mathrm{mmol})$ according to Procedure A. Purification by flash chromatography (eluent: pentane: ether $=10: 1$ ) provided the pure compound $\mathbf{3 j}$ (292 $\mathrm{mg}$, 99\%) as a colourless oil. $\mathrm{dr}>98: 2$.

${ }^{1}$ H NMR $\left(\mathrm{CDCl}_{3}, 300 \mathrm{MHz}\right): 7.40(\mathrm{~d}, J=8.8 \mathrm{~Hz}, 2 \mathrm{H}), 7.31(\mathrm{~d}, J=8.8 \mathrm{~Hz}, 2 \mathrm{H}), 5.81-5.87$ (m, $1 \mathrm{H}), 5.67-5.72(\mathrm{~m}, 1 \mathrm{H}), 1.93$ (s, $1 \mathrm{H}), 1.78-1.93$ (m, $2 \mathrm{H}), 1.59-1.77$ (m, $2 \mathrm{H}), 1.56$ (s, 3 $\mathrm{H}), 1.36-1.53(\mathrm{~m}, 1 \mathrm{H}), 0.98(\mathrm{~s}, 3 \mathrm{H}), 0.93-1.92(\mathrm{~m}, 1 \mathrm{H})$;

${ }^{13} \mathrm{C}$ NMR $\left(\mathrm{CDCl}_{3}, 75 \mathrm{MHz}\right): 144.2,131.5,130.3,130.1,129.0,120.5,78.0,42.7,30.6,25.0$, 24.9, 23.2, 19.4;

IR (neat): $3476(\mathrm{~m}), 2937$ (m), $1589(\mathrm{w}), 1486(\mathrm{~s}), 1368(\mathrm{~m}), 1076(\mathrm{~s}) \mathrm{cm}^{-1}$;

MS (EI, $70 \mathrm{ev}): \mathrm{m} / \mathrm{z}(\%)=276\left(\mathrm{M}^{+}-\mathrm{H}_{2} \mathrm{O}, 0.5\right), 201\left({ }^{81} \mathrm{Br}, 100\right), 199\left({ }^{79} \mathrm{Br}, 100\right), 185$ (9), 95 (21);

HRMS (EI): calcd. for $\mathrm{C}_{15} \mathrm{H}_{18} \mathrm{Br}\left(\mathrm{M}^{+}\left({ }^{79} \mathrm{Br}\right)-\mathrm{OH}\right)$ : 277.0592; found: $277.0589\left(\mathrm{M}^{+}\left({ }^{79} \mathrm{Br}\right)-\right.$ $\mathrm{OH})$.

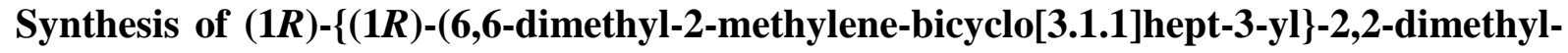
propan-1-ol (3k)

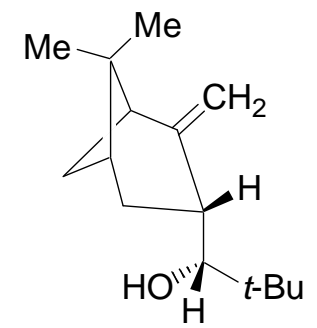

It was prepared from 2, 2-dimethyl-propionaldehyde $(86 \mathrm{mg}, 1.0 \mathrm{mmol})$ and ((6, 6-dimethyl bicyclo[3.1.1]hept-2-en-2-yl)methyl)zinc chloride (1d, $1.2 \mathrm{mmol}$ ) according to Procedure A. Purification by flash chromatography (eluent: pentane: ether $=3: 1$ ) provided the pure compound 3k $(200 \mathrm{mg}, 90 \%)$ as a colourless oil. $\mathrm{dr}>99: 1 .[\alpha]_{\mathrm{D}}{ }^{20}=+8.4\left(\mathrm{c}=0.5, \mathrm{CH}_{2} \mathrm{Cl}_{2}\right)$.

${ }^{1} \mathbf{H}$ NMR $\left(\mathrm{CDCl}_{3}, 600 \mathrm{MHz}\right): 4.94(\mathrm{~s}, 1 \mathrm{H}), 4.62(\mathrm{~s}, 1 \mathrm{H}), 3.08(\mathrm{t}, J=5.4 \mathrm{~Hz}, 1 \mathrm{H}), 2.57(\mathrm{t}, J=$ $7.5 \mathrm{~Hz}, 1 \mathrm{H}), 2.47(\mathrm{~d}, J=5.3 \mathrm{~Hz}, 1 \mathrm{H}), 2.33(\mathrm{~d}, J=5.0 \mathrm{~Hz}, 1 \mathrm{H}), 2.22-2.29(\mathrm{~m}, 1 \mathrm{H}), 2.06-$ $2.14(\mathrm{~m}, 1 \mathrm{H}), 1.95(\mathrm{q}, J=5.5 \mathrm{~Hz}, 1 \mathrm{H}), 1.63\left(\mathrm{dd}, J_{1}=13.8 \mathrm{~Hz}, J_{2}=4.4 \mathrm{~Hz}, 1 \mathrm{H}\right), 1.35(\mathrm{~d}, J=$ $10.2 \mathrm{~Hz}, 1 \mathrm{H}), 1.23$ (s, $3 \mathrm{H}), 0.91(\mathrm{~s}, 9 \mathrm{H}), 0.72$ (s, $3 \mathrm{H})$;

${ }^{13} \mathrm{C}$ NMR $\left(\mathrm{CDCl}_{3}, 150 \mathrm{MHz}\right): 153.7,111.9,83.6,51.7,41.4,40.5,37.3,36.4,33.5,25.77$, 25.75, 25.6, 21.6;

IR (neat): 3532 (m), 2920 (s), 2948 (s), 1630 (m), 1479 (m), 1458 (m), 1364 (s), 1076 (s) cm ;

MS (EI, $70 \mathrm{ev):} \mathrm{m/z} \mathrm{( \% )} \mathrm{=} 222\left(\mathrm{M}^{+}, 1\right), 189$ (5), 165 (8), 147 (9), 136 (39), 121 (30), 93 (100), 69 (23);

HRMS (EI): calcd. for $\mathrm{C}_{15} \mathrm{H}_{27} \mathrm{O}\left(\mathrm{M}^{+}+\mathrm{H}\right)$ : 223.2062; found: $223.2077\left(\mathrm{M}^{+}+\mathrm{H}\right)$. 
Synthesis of 3,5-dinitro-benzoic acid (1R)-\{(1R)-(6,6-dimethyl-2-methylenebicyclo[3.1.1]hept-3-yl\}-2,2-dimethyl-propyl ester (3k')
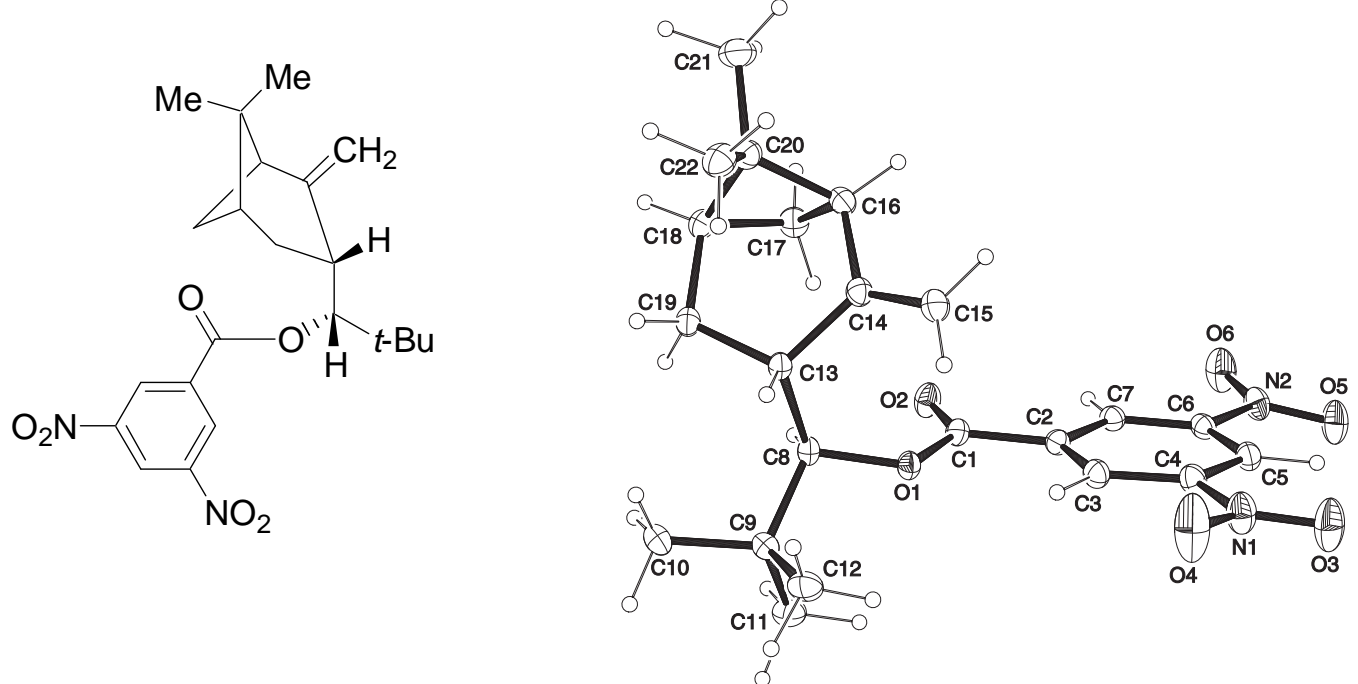

ORTEP representation of 10' with thermal ellipsoids set at $30 \%$ probability level (See the complete X-ray data at the end of the manuscript).

A solution of 1-(6, 6-dimethyl-2-methylene-bicyclo[3.1.1]hept-3-yl)-2,2-dimethyl-propan-1-

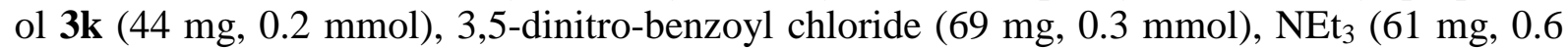
mmol) and DMAP $(10 \mathrm{~mol} \%)$ in $\mathrm{CH}_{2} \mathrm{Cl}_{2}(10 \mathrm{~mL})$ was stirred at room temperature for $12 \mathrm{~h}$. The reaction mixture was diluted with ether $(30 \mathrm{~mL})$ and then washed with water. The organic layer was dried over anhydrous $\mathrm{Na}_{2} \mathrm{SO}_{4}$, filtered, and concentrated. The residue was chromatographed on silica gel (eluent: pentane: ether $=8: 1)$, providing ' $3 \mathbf{k}$ ' $(71 \mathrm{mg}, 85 \%)$ as a white solid; mp.: $171.5-172.8^{\circ} \mathrm{C} .[\alpha]_{\mathrm{D}}{ }^{20}=-59.2\left(\mathrm{c}=0.5, \mathrm{CH}_{2} \mathrm{Cl}_{2}\right)$.

${ }^{1} \mathbf{H}$ NMR $\left(\mathrm{CDCl}_{3}, 300 \mathrm{MHz}\right): 9.17(\mathrm{t}, J=2.0 \mathrm{~Hz}, 1 \mathrm{H}), 9.11(\mathrm{~d}, J=2.0 \mathrm{~Hz}, 2 \mathrm{H}), 4.93(\mathrm{~d}, J=$ $8.8 \mathrm{~Hz}, 1 \mathrm{H}), 4.62-4.70(\mathrm{~m}, 2 \mathrm{H}), 2.92(\mathrm{t}, J=8.5 \mathrm{~Hz}, 1 \mathrm{H}), 2.06-2.34(\mathrm{~m}, 3 \mathrm{H}), 1.91-2.02(\mathrm{~m}, 1$ $\mathrm{H}), 1.69-1.82(\mathrm{~m}, 1 \mathrm{H}), 1.54(\mathrm{~d}, J=9.7 \mathrm{~Hz}, 1 \mathrm{H}), 1.19(\mathrm{~s}, 3 \mathrm{H}), 1.07(\mathrm{~s}, 9 \mathrm{H}), 0.70(\mathrm{~s}, 3 \mathrm{H})$;

${ }^{13}$ C NMR $\left(\mathrm{CDCl}_{3}, 75 \mathrm{MHz}\right): 161.6,150.5,148.6,134.7,129.4,122.0,112.7,87.5,51.9,40.9$, 40.5, 36.2, 35.4, 32.9, 26.5, 26.1, 25.5, 21.8;

IR (neat): 2912 (m), 1719 (s), 1628 (w), 1542 (vs), 1482(w), 1460 (w), 1339 (vs) cm cm $^{-1}$;

MS (EI, $70 \mathrm{ev}): \mathrm{m} / \mathrm{z}(\%)=401\left(\mathrm{M}^{+}-\mathrm{CH}_{3}, 0.5\right), 330$ (25), 194 (80), 105 (90), 91 (100);

Anal. Calcd for $\mathrm{C}_{22} \mathrm{H}_{28} \mathrm{~N}_{2} \mathrm{O}_{6}$ : C, 63.45; H, 6.78; N, 6.73; Found: C, 63.24; H, 6.56, N, 6.70.

Synthesis of $\left(2 R^{*}\right)-\left(3 S^{*}\right)-2-(4-b r o m o-p h e n y l)-3-p h e n y l-p e n t-4-e n-2-o l ~(4 a)$<smiles>C=C[C@@H](c1ccccc1)[C@](C)(O)c1ccc(Br)cc1</smiles>

It was prepared from 4-bromoacetophenone (199 $\mathrm{mg}, 1.0 \mathrm{mmol})$ and cinnamylzinc chloride (1e, $1.2 \mathrm{mmol}$ ) according to Procedure A. Purification by flash chromatography (eluent: pentane: ether $\left.=8: 2+1 \% \mathrm{Et}_{3} \mathrm{~N}\right)$ provided the pure compound $\mathbf{4 a}(315 \mathrm{mg}, 99 \%)$ as a colourless oil. dr > 99:1.

${ }^{1} \mathbf{H}$ NMR $\left(\mathrm{CDCl}_{3}, 300 \mathrm{MHz}\right): 7.42(\mathrm{~d}, J=8.5 \mathrm{~Hz}, 2 \mathrm{H})$, 7.05-7.32 (m, $\left.7 \mathrm{H}\right), 6.07-6.17$ (m, 1 H), $5.08(\mathrm{~d}, J=10.3 \mathrm{~Hz}, 1 \mathrm{H}), 4.96(\mathrm{~d}, J=17.1 \mathrm{~Hz}, 1 \mathrm{H}), 3.58(\mathrm{~d}, J=8.8 \mathrm{~Hz}, 1 \mathrm{H}), 2.00$ (br s, $1 \mathrm{H}), 1.43$ (s, $3 \mathrm{H})$.

${ }^{13} \mathrm{C}$ NMR $\left(\mathrm{CDCl}_{3}, 75 \mathrm{MHz}\right): 145.4,139.8,137.0,130.8,129.5,128.2,127.5,127.0,120.6$, 118.4, 76.0, 61.8, 28.4. 
IR (neat): 3560, 2974, 2924, 1638, 1598, 1486, 1452, 1394, 1084, 1008, 920, $743 \mathrm{~cm}^{-1}$. Spectral data matching those reported by Baba and collaborators. ${ }^{4}$

\section{Synthesis of $(2 S *)-(3 S *)-2,3-d i m e t h y l-4-p h e n y l-h e x-5-e n-3-o l(4 b)$}<smiles>C=C[C@H](c1ccccc1)[C@@](C)(O)C(C)C</smiles>

It was prepared from 3-methyl-butan-2-one $(86 \mathrm{mg}, 1.0 \mathrm{mmol})$ and cinnamylzinc chloride (1e, $1.2 \mathrm{mmol}$ ) according to Procedure A. Purification by flash chromatography (eluent: pentane: ether $\left.=8: 2+1 \% \mathrm{Et}_{3} \mathrm{~N}\right)$ provided the pure compound $\mathbf{4 b}(196 \mathrm{mg}, 96 \%)$ as a colourless oil. $\mathrm{dr}>$ 99:1.

${ }^{1} \mathbf{H}$ NMR $\left(\mathrm{CDCl}_{3}, 300 \mathrm{MHz}\right): 7.22-7.38(\mathrm{~m}, 5 \mathrm{H}), 6.39(\mathrm{dt}, J=17.2 \mathrm{~Hz}, J=9.9 \mathrm{~Hz}, 1 \mathrm{H}), 5.19$ $(\mathrm{dd}, J=10.2 \mathrm{~Hz}, J=1.9 \mathrm{~Hz}, 1 \mathrm{H}), 5.13(\mathrm{ddd}, J=17.2 \mathrm{~Hz}, J=1.8 \mathrm{~Hz}, J=0.7 \mathrm{~Hz}, 1 \mathrm{H}), 3.46$ $(\mathrm{d}, J=9.6 \mathrm{~Hz}, 1 \mathrm{H}), 2.00$ (hept, $J=6.8 \mathrm{~Hz}, 1 \mathrm{H}), 1.40$ (br s, $1 \mathrm{H}), 1.02(\mathrm{~d}, J=6.9 \mathrm{~Hz}, 3 \mathrm{H})$, $0.96(d, \mathrm{~J}=6.8 \mathrm{~Hz}, 3 \mathrm{H}), 0.91(s, 3 \mathrm{H})$.

${ }^{13} \mathrm{C}$ NMR $\left(\mathrm{CDCl}_{3}, 75 \mathrm{MHz}\right): 142.1,138.1,129.6,128.5,126.7,117.1,76.3,57.9,34.3,20.4$, $17.8,17.1$.

IR (neat): 3480, 3074, 3027, 2976, 2962, 2877, 1636, 1601, 1491, 1470, 1452, 1387, 1376, $1156,1080,1001,912,734 \mathrm{~cm}^{-1}$;

MS (EI, 70 ev): m/z (\%) = 161 (3), 119 (8), 118 (87), 117 (28), 115 (14), 91 (9), 87 (100), 69 (27).

HRMS (EI): calcd. for $\left[\mathrm{C}_{14} \mathrm{H}_{20} \mathrm{O}-\mathrm{OH}\right]^{+}:$187.1487; found: 187.1466 .

Synthesis of $\left(2 S^{*}\right)-\left(3 S^{*}\right)-2-c y c l o p r o p y l-3-p h e n y l-p e n t-4-e n-2-o l(4 c)$<smiles>C=CC(c1ccccc1)C(C)(O)C1CC1</smiles>

It was prepared from 1-cyclopropyl-ethanone $(85 \mathrm{mg}, 1.0 \mathrm{mmol})$ and cinnamylzinc chloride (1e, $1.2 \mathrm{mmol}$ ) according to Procedure A. Purification by flash chromatography (eluent: pentane: ether $\left.=8: 2+1 \% \mathrm{Et}_{3} \mathrm{~N}\right)$ provided the pure compound $\mathbf{4 c}(188 \mathrm{mg}, 93 \%)$ as a colourless oil. dr $>$ 99:1.

${ }^{1} \mathbf{H}$ NMR $\left(\mathrm{CDCl}_{3}, 300 \mathrm{MHz}\right): 7.23-7.39(\mathrm{~m}, 5 \mathrm{H}), 6.44(\mathrm{dt}, J=16.9 \mathrm{~Hz}, J=9.9 \mathrm{~Hz}, 1 \mathrm{H})$, 5.15-5.26 (m, $2 \mathrm{H}), 3.44(\mathrm{~d}, J=9.4 \mathrm{~Hz}, 1 \mathrm{H}), 1.33(\mathrm{~s}, 1 \mathrm{H}), 1.11(\mathrm{~s}, 3 \mathrm{H}), 0.84-0.95(\mathrm{~m}, 1 \mathrm{H})$, $0.28-0.47(\mathrm{~m}, 4 \mathrm{H})$.

${ }^{13} \mathrm{C}$ NMR $\left(\mathrm{CDCl}_{3}, 75 \mathrm{MHz}\right): 140.9,137.8,129.4,128.1,126.6,117.7,72.4,62.1,24.3,19.5$, $1.1,0.8$.

IR (neat): 3570, 3475, 3082, 3006, 2977, 2932, 1636, 1601, 1493, 1452, 1418, 1372, 1307, $1138,1020,915,734 \mathrm{~cm}^{-1}$.

MS (EI, 70 ev): m/z (\%) = 128 (1), 119 (3), 118 (29), 117 (13), 115 (10), 86 (3), 85 (52), 67 (4), 43 (100).

HRMS (EI): calcd. for $\left[\mathrm{C}_{14} \mathrm{H}_{18} \mathrm{O}-\mathrm{H}_{2} \mathrm{O}\right]^{+}:$184.1252; found: 184.1264 .

Note: The same reaction carried out at $0{ }^{\circ} \mathrm{C}$ afforded $4 \mathbf{c}$ in $92 \%$ yield and 92: 8 diastereoselectivity.

\footnotetext{
${ }^{4}$ Yasuda, M.; Hirata, K.; Nishino, M.; Yamamoto, A.; Baba, A. J. Am. Chem. Soc. 2002, 124, 13442-13447.
} 
Synthesis of (2S*)-(3S*)-2-cyclohexyl-3-phenyl-pent-4-en-2-ol (4d)<smiles>C=C[C@@H](c1ccccc1)[C@@](C)(O)C1CCCCC1</smiles>

It was prepared from 1-cyclohexyl-ethanone $(127 \mathrm{mg}, 1.0 \mathrm{mmol})$ and cinnamylzinc chloride (1e, $1.2 \mathrm{mmol}$ ) according to Procedure A. Purification by flash chromatography (eluent: pentane: ether $\left.=8: 2+1 \% \mathrm{Et}_{3} \mathrm{~N}\right)$ provided the pure compound $\mathbf{4 d}(231 \mathrm{mg}, 95 \%)$ as a colourless oil. $\mathrm{dr}>99: 1$.

${ }^{1} \mathbf{H}$ NMR $\left(\mathrm{CDCl}_{3}, 300 \mathrm{MHz}\right): 7.21-7.37(\mathrm{~m}, 5 \mathrm{H}), 6.37(\mathrm{dt}, J=17.2 \mathrm{~Hz}, J=9.9 \mathrm{~Hz}, 1 \mathrm{H}), 5.18$ $(\mathrm{dd}, J=10.2 \mathrm{~Hz}, J=1.9 \mathrm{~Hz}, 1 \mathrm{H}), 5.10(\mathrm{dd}, J=17.2 \mathrm{~Hz}, J=1.6 \mathrm{~Hz}, 1 \mathrm{H}), 3.47(\mathrm{~d}, J=9.7 \mathrm{~Hz}$, $1 \mathrm{H}), 1.58-2.06$ (m, $6 \mathrm{H}), 0.94-1.58(\mathrm{~m}, 6 \mathrm{H}), 0.91$ (s, $3 \mathrm{H})$.

${ }^{13} \mathbf{C}$ NMR $\left(\mathrm{CDCl}_{3}, 75 \mathrm{MHz}\right): 141.9,137.9,129.4,128.2,126.4,116.8,75.6,57.3,44.5,27.8$, 26.7, 26.7, 21.5.

IR (neat): 3477, 3072, 3027, 2976, 2903, 2852, 1635, 1600, 1490, 1451, 1378, 1062, 909, 746, $701 \mathrm{~cm}^{-1}$.

MS (EI, $70 \mathrm{ev}): \mathrm{m} / \mathrm{z}(\%)=161$ (4), 128 (6), 127 (68), 119 (9), 118 (100), 117 (25), 115 (13), 109 (37), 83 (40), 43 (30).

HRMS (EI): calcd. for $\left[\mathrm{C}_{17} \mathrm{H}_{24} \mathrm{O}-\mathrm{H}_{2} \mathrm{O}\right]^{+}:$:226.1721; found: 226.1716 .

Synthesis of (2S*)-(3S*)-1,1,1-trifluoro-2-methyl-3-phenyl-pent-4-en-2-ol (4e)<smiles>C=CC(c1ccccc1)C(O)(O)C(F)(F)F</smiles>

It was prepared from 1,1,1-trifluoro-propan-2-one $(111 \mathrm{mg}, 1.0 \mathrm{mmol})$ and cinnamylzinc chloride (1e, $1.2 \mathrm{mmol}$ ) according to Procedure A. Purification by flash chromatography (eluent: pentane: ether $\left.=9: 1+1 \% \mathrm{Et}_{3} \mathrm{~N}\right)$ provided the pure compound $4 \mathbf{e}(177 \mathrm{mg}, 77 \%)$ as a colourless oil. dr $>$ 99:1.

${ }^{1} \mathbf{H}$ NMR $\left(\mathrm{CDCl}_{3}, 300 \mathrm{MHz}\right):$ 7.24-7.39 (m, $\left.5 \mathrm{H}\right), 6.29-6.44(\mathrm{~m}, 1 \mathrm{H}), 5.24(\mathrm{dd}, J=10.2 \mathrm{~Hz}, J$ $=1.3 \mathrm{~Hz}, 1 \mathrm{H}), 5.18(\mathrm{ddd}, J=17.0 \mathrm{~Hz}, J=1.4 \mathrm{~Hz}, J=1.1 \mathrm{~Hz}, 1 \mathrm{H}), 3.65(\mathrm{~d}, J=9.0 \mathrm{~Hz}, 1 \mathrm{H})$, $2.21(\mathrm{br} \mathrm{s}, 1 \mathrm{H}), 1.28(\mathrm{q}, J=1.1 \mathrm{hz}, 3 \mathrm{H})$.

${ }^{13} \mathbf{C ~ N M R}\left(\mathrm{CDCl}_{3}, 75 \mathrm{MHz}\right): 138.9,135.6,129.2,128.5,127.3,126.1(\mathrm{q}, J=286.9 \mathrm{~Hz})$, 118.6, $76.8(\mathrm{q}, J=26.6 \mathrm{~Hz}), 55.1,20.6(\mathrm{~m})$.

IR (neat): 3452, 3083, 2982, 2936, 1639, 1602, 1492, 1455, 1384, 1251, 1176, 1120, 1091, 996, 924, 751, $706 \mathrm{~cm}^{-1}$.

MS (EI, 70 ev): m/z (\%) = 230 (5), 118 (17), 117 (100), 116 (9), 115 (37), 91 (17), 65 (4), 43 (12).

HRMS (EI): calcd. for $\left[\mathrm{C}_{12} \mathrm{H}_{13} \mathrm{~F}_{3} \mathrm{O}\right]^{+}:$:230.0918; found: 230.0895 .

Synthesis of (2S*)-(3S*)-2-isopropyl-2-methyl-3-phenyl-tetrahydro-furan (5)<smiles>CC1(C)OCC[C@@H]1c1ccccc1</smiles>

$\mathrm{BH}_{3} \cdot \mathrm{Me}_{2} \mathrm{~S}$ (1.8 mL, $19 \mathrm{mmol}, 3$ equiv)) was added at room temperature to a solution of homoallylic alcohol $\mathbf{4 b}(1.2 \mathrm{~g}, 6.0 \mathrm{mmol})$ in dry THF $(20 \mathrm{~mL})$. The resulting solution was stirred at room temperature under nitrogen for $4 \mathrm{~h}$, then it was quenched with water $(7 \mathrm{~mL})$ at $0{ }^{\circ} \mathrm{C}$. Sodium perborate ( $8.5 \mathrm{~g}, 55 \mathrm{mmol}, 9$ equiv) and $\mathrm{NaOH}(2.2 \mathrm{~g}, 55 \mathrm{mmol}, 9$ equiv) were 
subsequently added, and the reaction mixture was heated to $45^{\circ} \mathrm{C}$ for $4 \mathrm{~h}$. The phases were separated and the aqueous phase was extracted with $\mathrm{Et}_{2} \mathrm{O}$. The combined organic phases were dried over $\mathrm{MgSO}_{4}$, and concentrated in vacuo. Purification by flash chromatography (eluent: ether $\left.+1 \% \mathrm{Et}_{3} \mathrm{~N}\right)$ provided $\left(4 S^{*}\right)-\left(3 S^{*}\right)-4,5$-dimethyl-3-phenyl-hexane-1,4-diol $(1.1 \mathrm{~g}, 84 \%)$ as a colourless oil. $\mathrm{dr}>99: 1$.

${ }^{1} \mathbf{H}$ NMR $\left(\mathrm{CDCl}_{3}, 300 \mathrm{MHz}\right): 7.21-7.36(\mathrm{~m}, 5 \mathrm{H}), 3.48-3.60(\mathrm{~m}, 1 \mathrm{H}), 3.29-3.42(\mathrm{~m}, 1 \mathrm{H}), 2.95$ $(\mathrm{dd}, J=11.1 \mathrm{~Hz}, J=3.6 \mathrm{~Hz}, 1 \mathrm{H}), 2.11-2.25(\mathrm{~m}, 1 \mathrm{H}), 1.89-2.09(\mathrm{~m}, 2 \mathrm{H}), 1.44(\mathrm{br} \mathrm{s}, 2 \mathrm{H})$, $1.02(\mathrm{~d}, J=6.7 \mathrm{~Hz}, 3 \mathrm{H}), 0.95(d, \mathrm{~J}=6.9 \mathrm{~Hz}, 3 \mathrm{H}), 0.91(\mathrm{~s}, 3 \mathrm{H})$.

${ }^{13} \mathrm{C}$ NMR $\left(\mathrm{CDCl}_{3}, 75 \mathrm{MHz}\right): 141.8,129.6,128.2,126.6,76.4,61.5,49.2,34.0,31.8,20.7$, $17.8,17.0$.

IR (neat): 3317, 2966, 2912, 2877, 1601, 1451, 1364, 1158, 1054, 1031, 931, 733, $700 \mathrm{~cm}^{-1}$. MS (EI, $70 \mathrm{ev):} \mathrm{m/z} \mathrm{( \% )} \mathrm{=} 189$ (2), 161 (32), 136 (16), 118 (100), 117 (31), 105 (18), 91 (16), 87 (58), 69 (11), 43 (26).

HRMS (EI): calcd. for $\left[\mathrm{C}_{14} \mathrm{H}_{22} \mathrm{O}_{2}\right]^{+}: 222,1620$; found: 222.1607 .

Mesyl chloride $\left(0.5 \mathrm{~mL}, 6.5 \mathrm{mmol}, 1.5\right.$ equiv) was added at $0{ }^{\circ} \mathrm{C}$ to a solution of the diol $(0.9$ $\mathrm{g}, 4.1 \mathrm{mmol}), \mathrm{Et}_{3} \mathrm{~N}$ (1.1 mL, $8 \mathrm{mmol}, 2.0$ equiv) and DMAP $(30 \mathrm{mg})$ in $\mathrm{CH}_{2} \mathrm{Cl}_{2}(15 \mathrm{~mL})$. After $90 \mathrm{~min}$, water was added and the aqueous phase extracted with $\mathrm{CH}_{2} \mathrm{Cl}_{2}$. The combined organic phases were dried over $\mathrm{MgSO}_{4}$, and concentrated in vacuo. Purification by flash chromatography (eluent: pentane: ether $=98: 2)$ the pure tetrahydrofuran $\mathbf{5}(571 \mathrm{mg}, 69 \%)$ as a colourless oil. dr $>$ 99:1.

${ }^{1} \mathbf{H}$ NMR $\left(\mathrm{CDCl}_{3}, 600 \mathrm{MHz}\right): 7.19-7.32(\mathrm{~m}, 5 \mathrm{H}), 4.06-4.11(\mathrm{~m}, 1 \mathrm{H}), 3.87(\mathrm{q}, J=8.1 \mathrm{~Hz}, 1$ H), 2.25-2.40 (m, $2 \mathrm{H}), 1.88$ (hept, $J=6.8 \mathrm{~Hz}, 1 \mathrm{H}), 1.00$ (d, $J=6.8 \mathrm{~Hz}, 3 \mathrm{H}), 0.95$ (d, $J=6.9$ $\mathrm{Hz}, 3 \mathrm{H}), 0.79$ (s, $3 \mathrm{H})$.

${ }^{13} \mathbf{C}$ NMR $\left(\mathrm{CDCl}_{3}, 75 \mathrm{MHz}\right): 142.4,128.6,128.1,126.2,87.3,65.5,50.3,35.5,33.7,19.0$, $18.3,17.1$.

IR (neat): 3029, 2964, 2875, 1603, 1495, 1453, 1386, 1372, 1082, 1052, 852, $767 \mathrm{~cm}^{-1}$.

MS (EI, 70 ev): m/z (\%) = 167 (3), 162 (6), 119 (10), 118 (100), 117 (59), 91 (13), 65 (2), 43 (17).

HRMS (EI): calcd. for $\left[\mathrm{C}_{14} \mathrm{H}_{20} \mathrm{O}+\mathrm{H}\right]^{+}:$205.1592; found: 205.1584 .

\section{Synthesis of (2R*)-(3S)-2-methyl-3-phenyl-2-trifluoromethyl-tetrahydro-furan (6)}<smiles>C[C@]1(C(F)(F)F)OCC[C@@H]1c1ccccc1</smiles>

$\mathrm{BH}_{3} \cdot \mathrm{Me}_{2} \mathrm{~S}$ (1.8 mL, $19 \mathrm{mmol}, 3$ equiv)) was added at room temperature to a solution of homoallylic alcohol $4 \mathrm{e}(1.4 \mathrm{~g}, 6.0 \mathrm{mmol})$ in dry THF $(20 \mathrm{~mL})$. The resulting solution was stirred at room temperature under nitrogen for $4 \mathrm{~h}$, then it was quenched with water $(7 \mathrm{~mL})$ at $0{ }^{\circ} \mathrm{C}$. Sodium perborate $(8.5 \mathrm{~g}, 55 \mathrm{mmol}, 9$ equiv) and $\mathrm{NaOH}(2.2 \mathrm{~g}, 55 \mathrm{mmol}, 9$ equiv) were subsequently added, and the reaction mixture was heated to $45^{\circ} \mathrm{C}$ for $4 \mathrm{~h}$. The phases were separated and the aqueous phase was extracted with $\mathrm{Et}_{2} \mathrm{O}$. The combined organic phases were dried over $\mathrm{MgSO}_{4}$, and concentrated in vacuo. Purification by flash chromatography (eluent: pentane: ether $\left.=6: 4+1 \% \mathrm{Et}_{3} \mathrm{~N}\right)$ provided $\left(4 R^{*}\right)-\left(3 S^{*}\right)-5,5,5$-trifluoro-4-methyl-3-phenylpentane-1,4-diol (554 $\mathrm{mg}, 37 \%)$ as a white solid. $\mathrm{dr}>99: 1$.

${ }^{1} \mathbf{H}$ NMR $\left(\mathrm{CDCl}_{3}, 300 \mathrm{MHz}\right): 7.20-7.34(\mathrm{~m}, 5 \mathrm{H}), 3.57$ (quint $\left., J=5.3 \mathrm{~Hz}, 1 \mathrm{H}\right), 3.22-3.41(\mathrm{~m}$, $1 \mathrm{H}), 3.18(\mathrm{dd}, J=10.1 \mathrm{~Hz}, J=3.8 \mathrm{~Hz}, 1 \mathrm{H}), 2.11-2.25(\mathrm{~m}, 1 \mathrm{H}), 2.16-2.50(\mathrm{~m}, 3 \mathrm{H}), 2.01-$ $2.13(\mathrm{~m}, 1 \mathrm{H}), 1.27$ (br s, $3 \mathrm{H})$.

${ }^{13} \mathbf{C ~ N M R}\left(\mathrm{CDCl}_{3}, 75 \mathrm{MHz}\right): 139.1,129.5$ (br), 128.4, 127.3, 126.5 (q, J = $\left.287.2 \mathrm{~Hz}\right), 75.9$ (q, $\mathrm{J}=26.4 \mathrm{hz}), 60.6,47.1,32.6,19.8(\mathrm{~m})$. 
IR (neat): 3335, 3119, 2888, 1604, 1488, 1455, 1297, 1242, 1188, 1168, 1132, 1037, 706, 669 $\mathrm{cm}^{-1}$.

MS (EI, $70 \mathrm{ev):} \mathrm{m/z} \mathrm{( \% )} \mathrm{=} 137$ (2), 136 (23), 135 (16), 118 (37), 117 (19), 106 (9), 105 (100), 91 (51), 43 (10).

HRMS (EI): calcd. for $\left[\mathrm{C}_{12} \mathrm{H}_{15} \mathrm{~F}_{3} \mathrm{O}_{2}-\mathrm{H}_{2} \mathrm{O}\right]^{+}:$230.0918; found: 230.0906 .

Mesyl chloride $\left(0.16 \mathrm{~mL}, 1.5\right.$ equiv) was added at $0{ }^{\circ} \mathrm{C}$ to a solution of the diol $(345 \mathrm{mg}, 1.4$ mmol), $\mathrm{Et}_{3} \mathrm{~N}\left(0.4 \mathrm{~mL}, 2.0\right.$ equiv) and DMAP $(8 \mathrm{mg})$ in $\mathrm{CH}_{2} \mathrm{Cl}_{2}(3 \mathrm{~mL})$ and $\mathrm{Et}_{2} \mathrm{O}(3 \mathrm{~mL})$. After $40 \mathrm{~min}$, water was added and the aqueous phase extracted with $\mathrm{Et}_{2} \mathrm{O}$. The combined organic phases were dried over $\mathrm{MgSO}_{4}$, and concentrated in vacuo. Purification by flash chromatography (eluent: pentane: ether $=98: 2)$ the pure tetrahydrofuran $6(151 \mathrm{mg}, 47 \%)$ as a colourless oil. dr $>$ 99:1.

${ }^{1} \mathbf{H}$ NMR $\left(\mathrm{CDCl}_{3}, 300 \mathrm{MHz}\right): 7.23-7.40(\mathrm{~m}, 5 \mathrm{H}), 4.19-4.29(\mathrm{~m}, 1 \mathrm{~h}), 4.04$ (q, $J=8.0 \mathrm{~Hz}, 1$ $\mathrm{H}), 3.73(\mathrm{t}, J=8.6 \mathrm{~Hz}, 1 \mathrm{H}), 2.37-2.48(\mathrm{~m}, 2 \mathrm{H}), 0.99(\mathrm{q}, J=1.1 \mathrm{~Hz}, 3 \mathrm{H})$.

${ }^{13} \mathbf{C ~ N M R}\left(\mathrm{CDCl}_{3}, 75 \mathrm{MHz}\right): 138.4,128.8,128.4,127.3,127.0(\mathrm{q}, J=286.2 \mathrm{~Hz}), 84.2(\mathrm{q}, J=$ $27.4 \mathrm{~Hz}), 68.6,48.3,32.8,18.5(\mathrm{~m})$.

IR (neat): 3034, 2994, 2952, 2885, 1604, 1495, 1456, 1383, 1286, 1208, 1179, 1117, 1084, $1044,916,883,773,700 \mathrm{~cm}^{-1}$.

MS (EI, 70 ev): 231 (4), 230 (29), 161 (11), 118 (100), 117 (96), 115 (13), 91 (17), 43 (7).

HRMS (EI): calcd. for $\left[\mathrm{C}_{12} \mathrm{H}_{13} \mathrm{~F}_{3} \mathrm{O}\right]^{+}: 230.0918$; found: 230.0914 . 

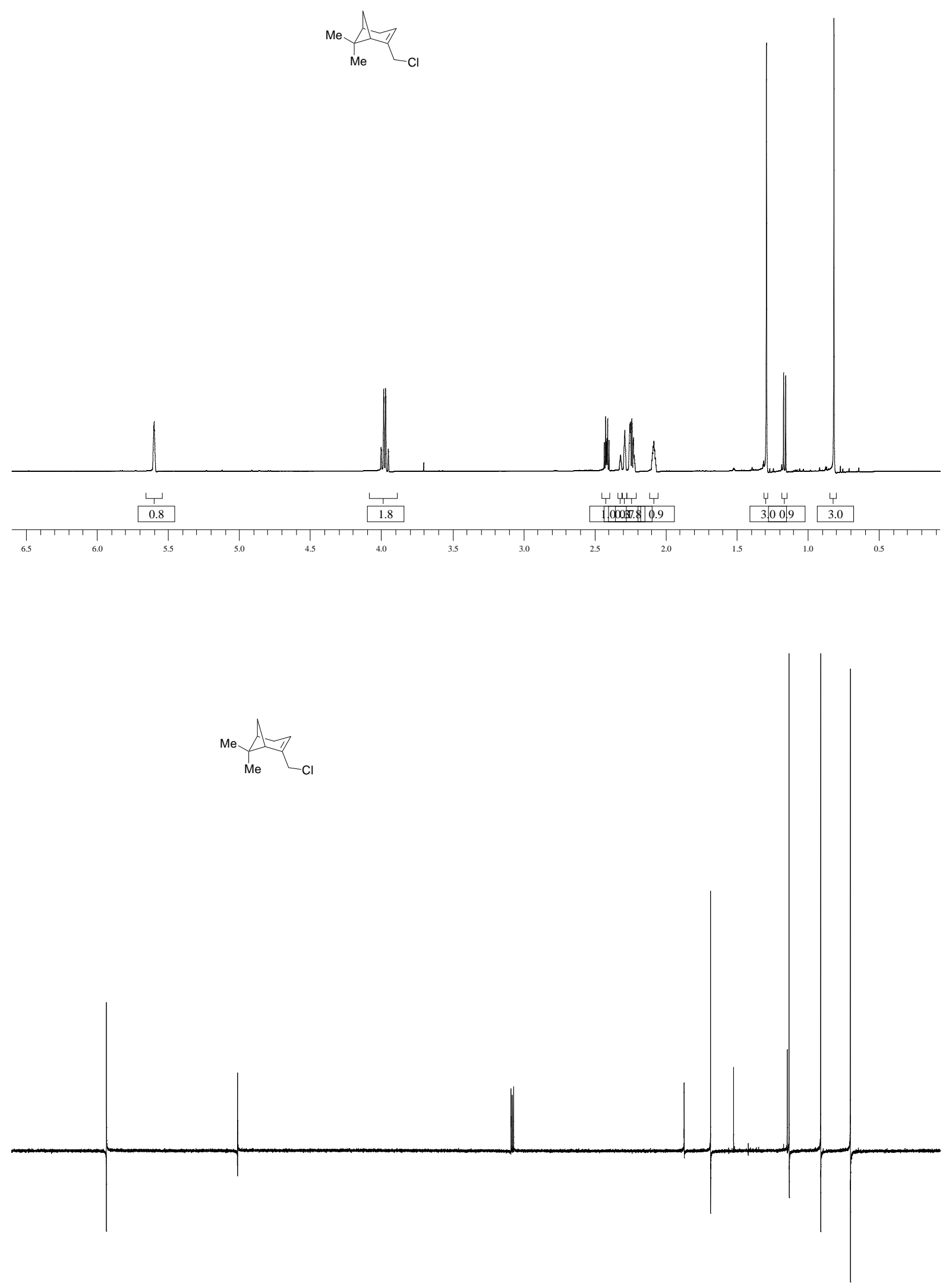

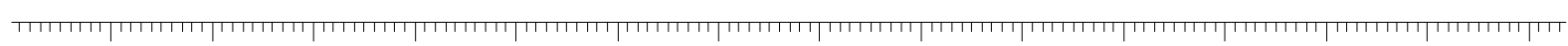

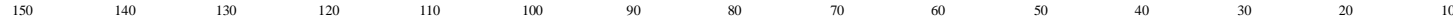



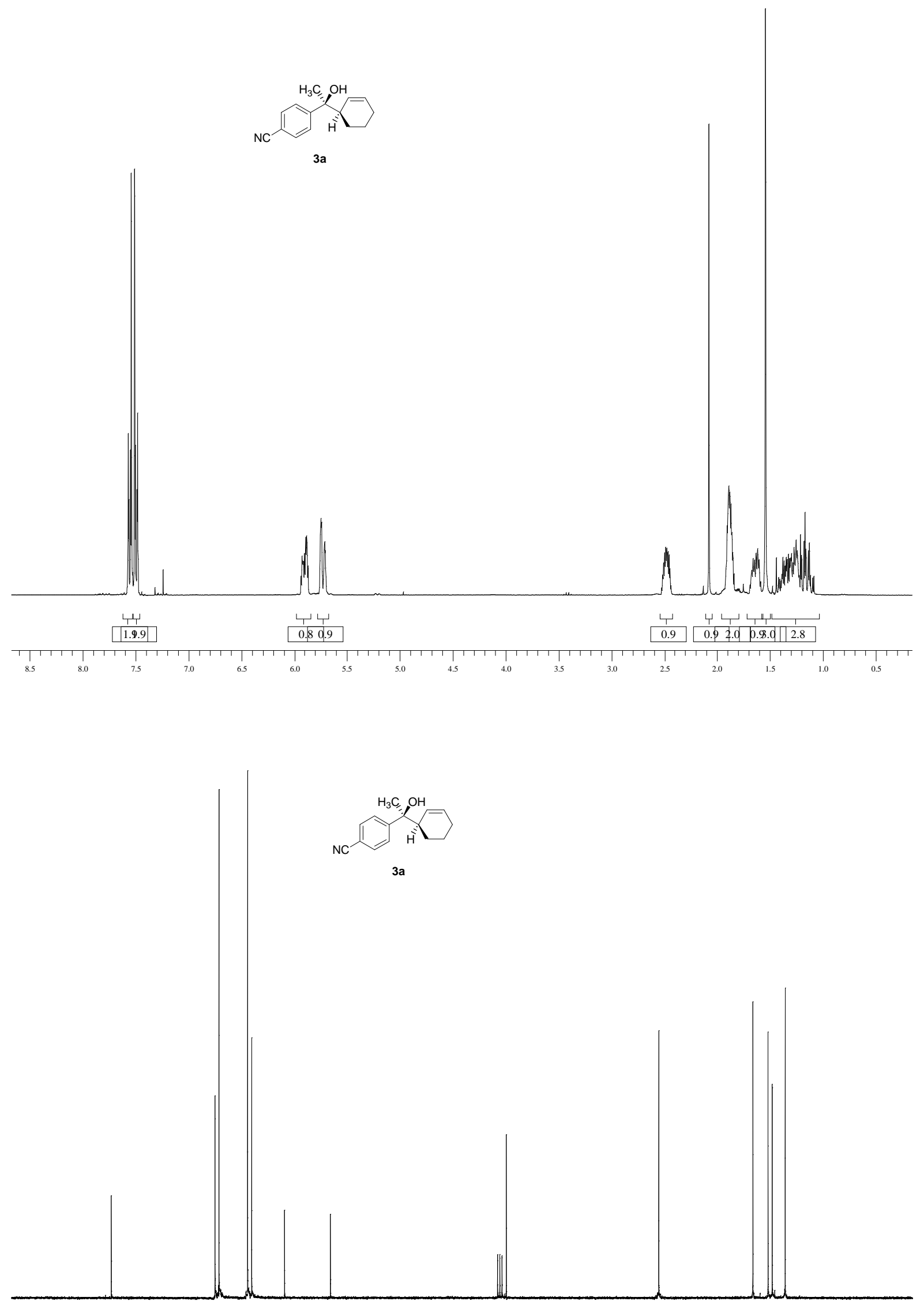

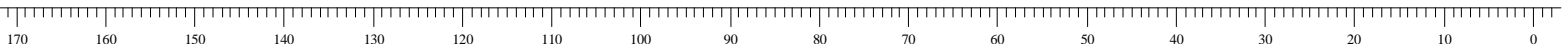




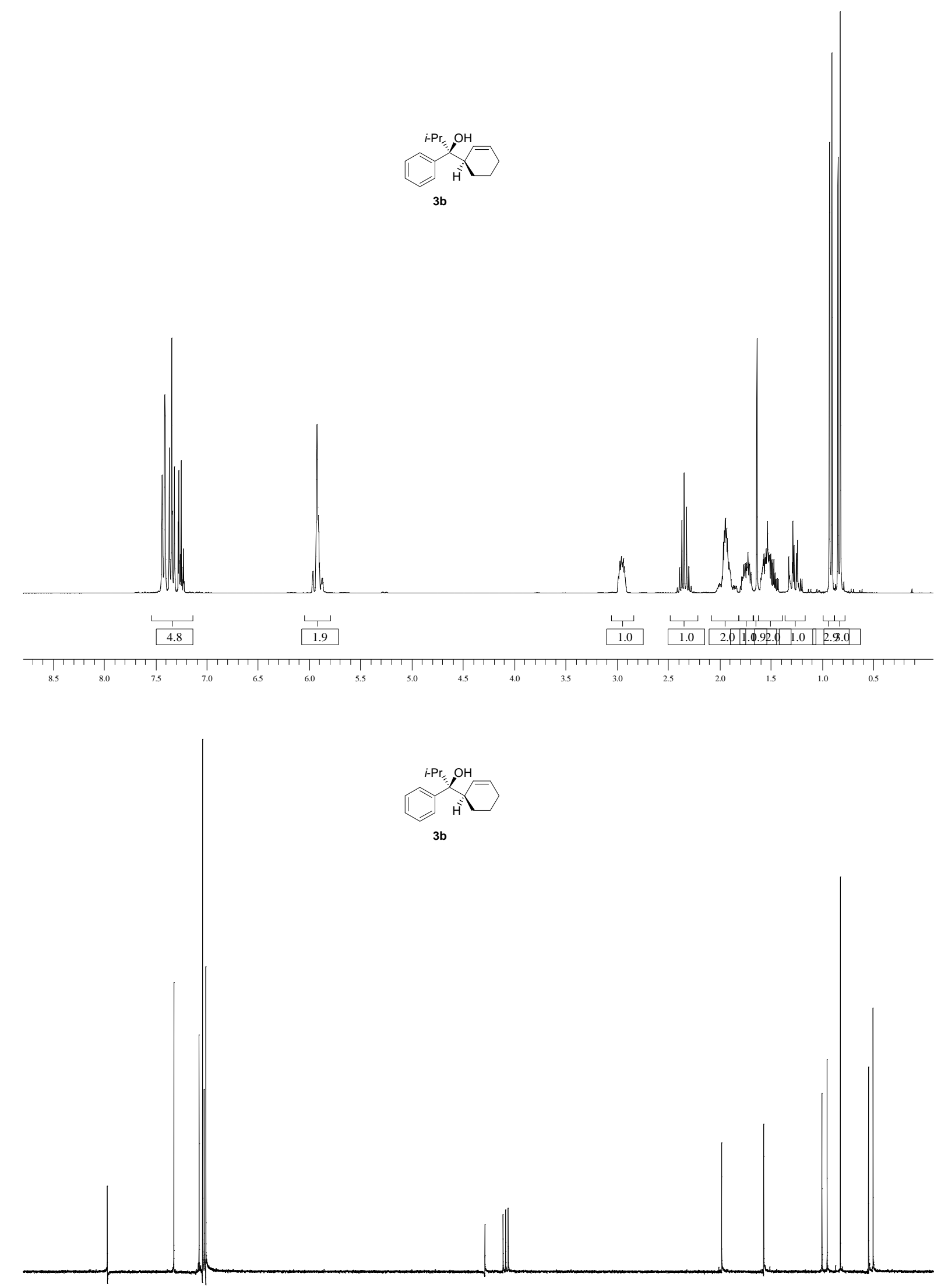

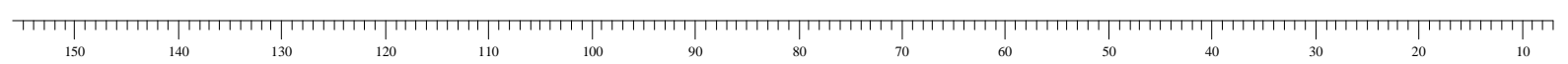


$\left.\right|_{3 c} ^{\mathrm{CH}}$
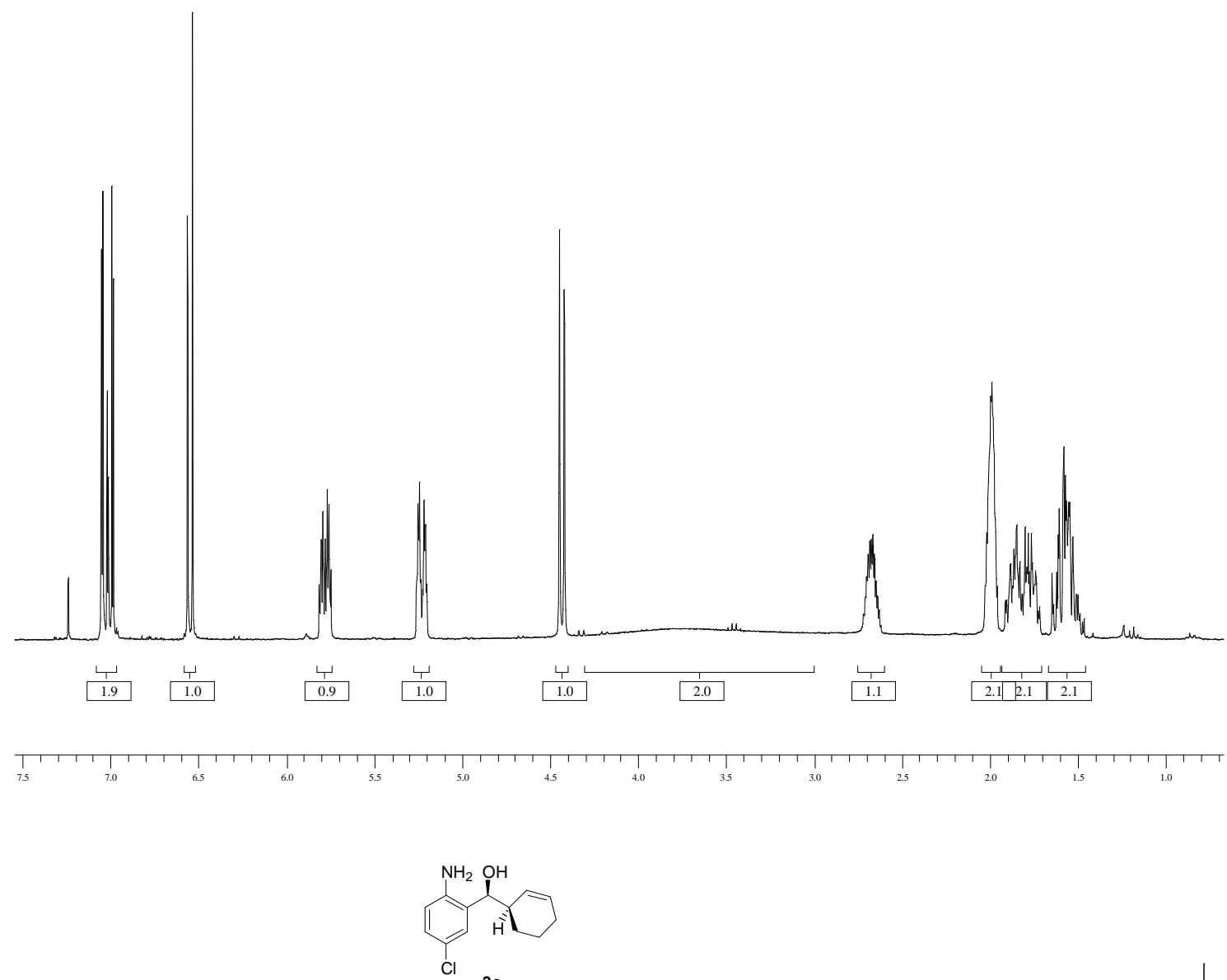

$3 c$

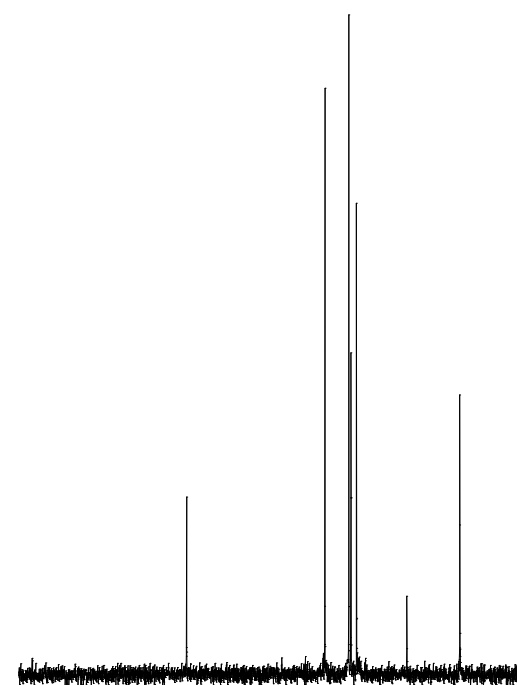

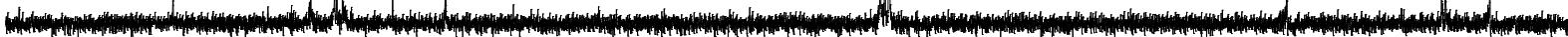




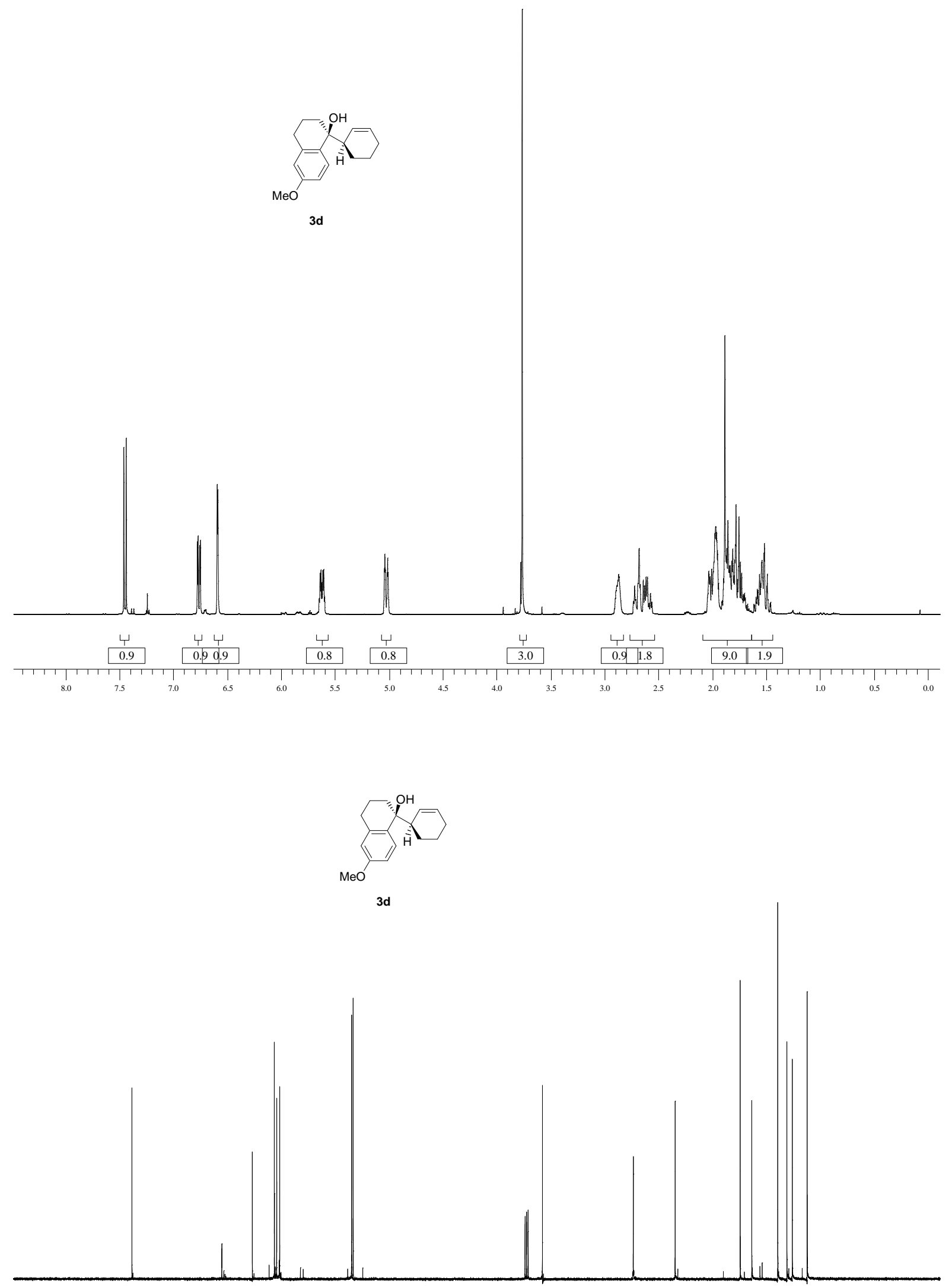

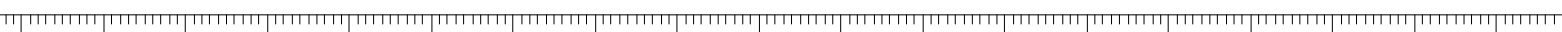

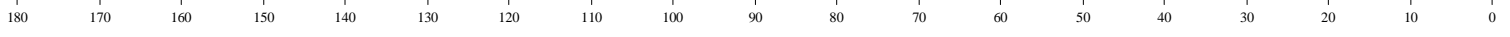



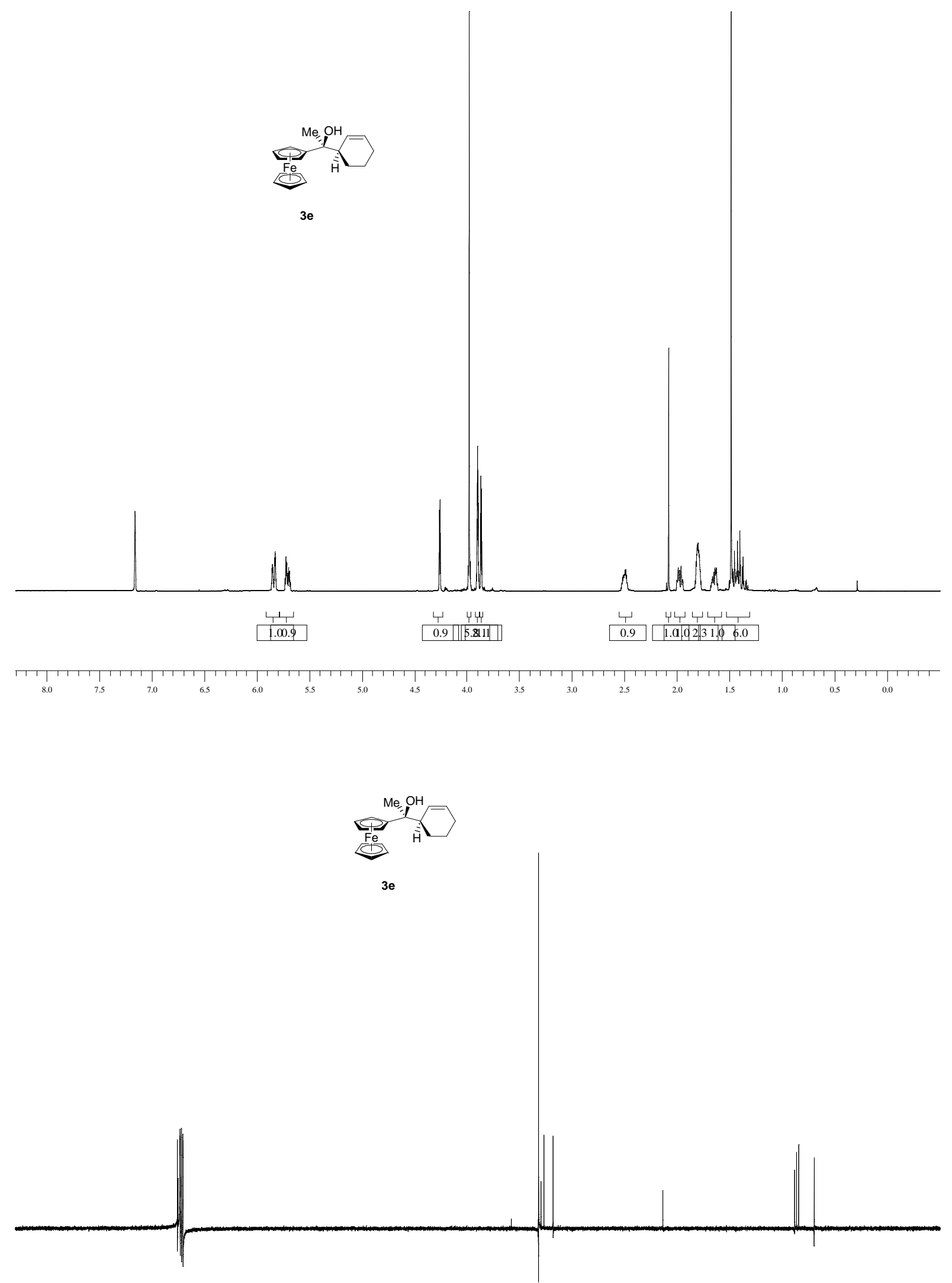

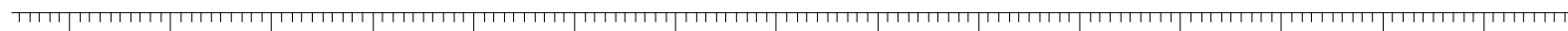

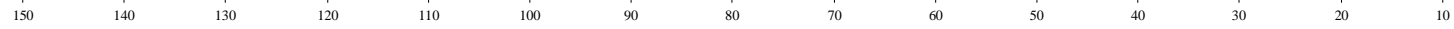



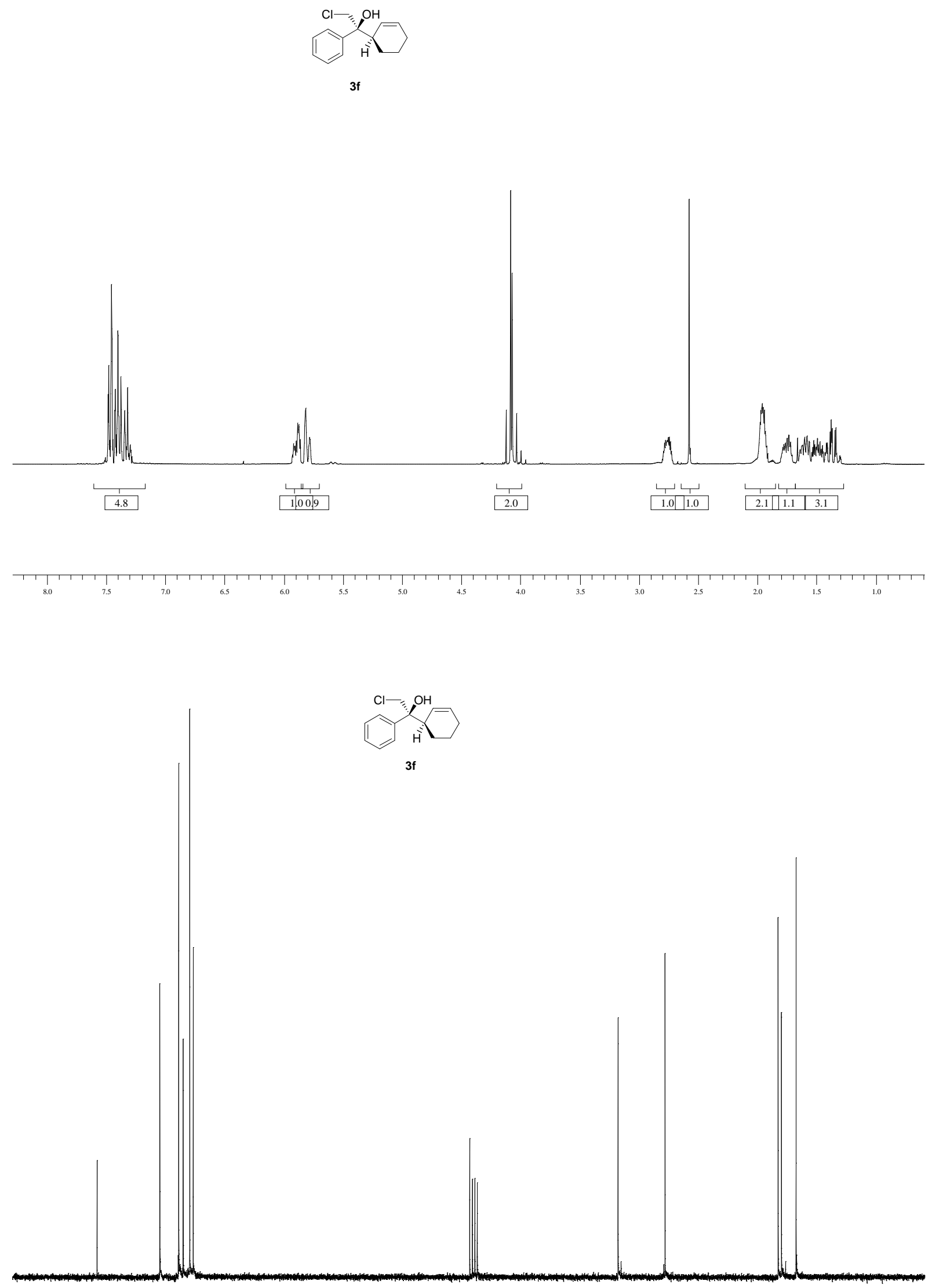

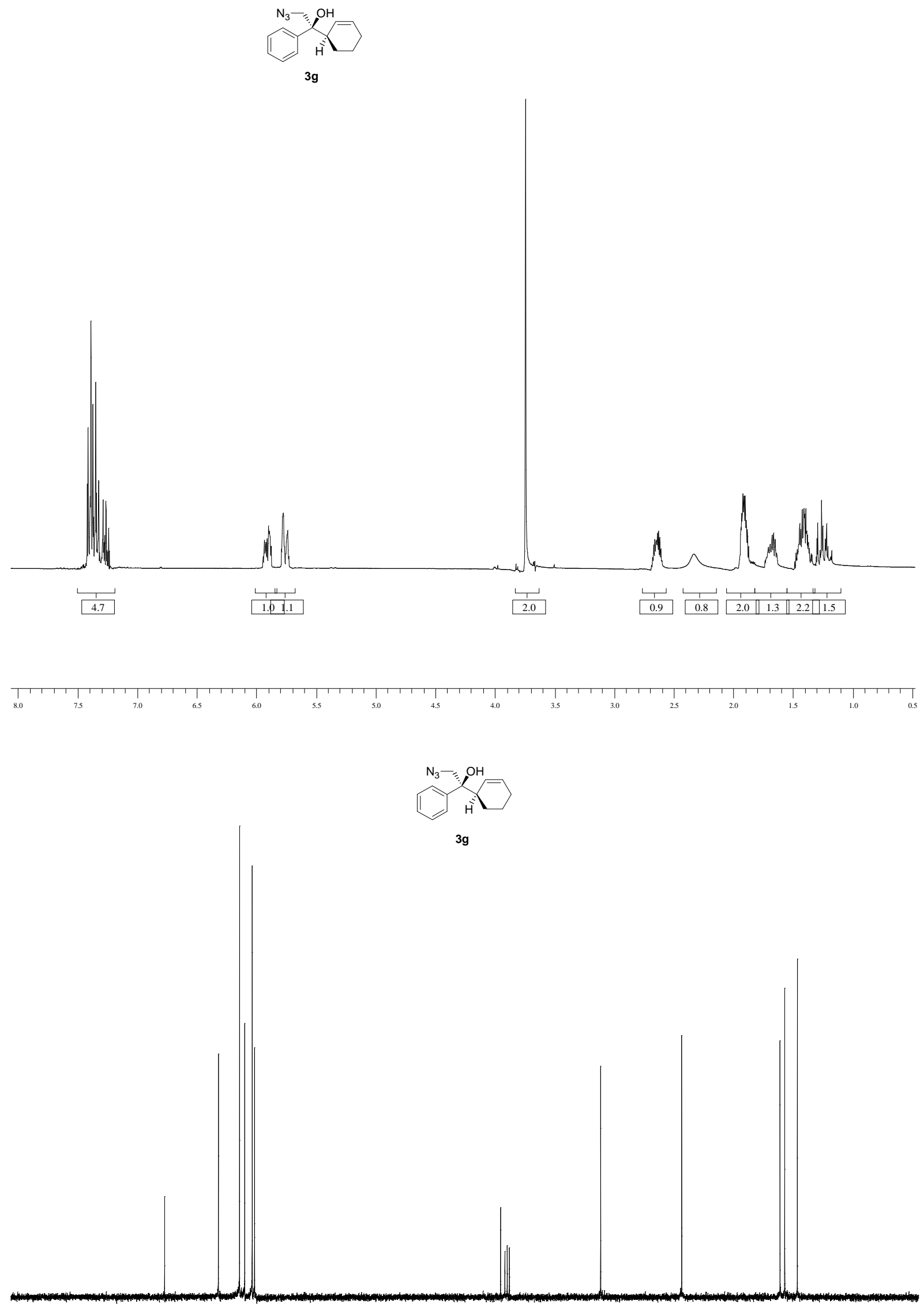

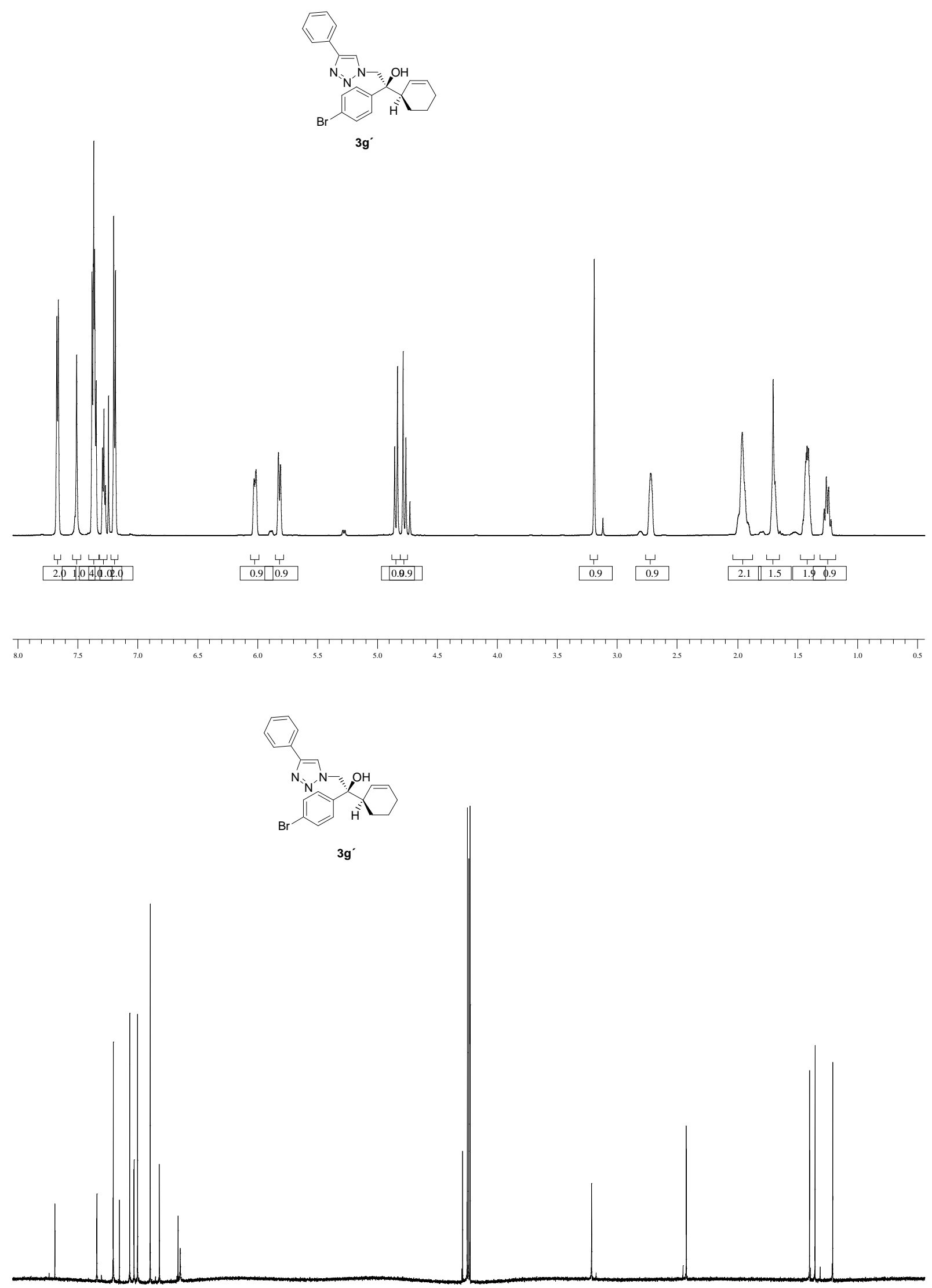

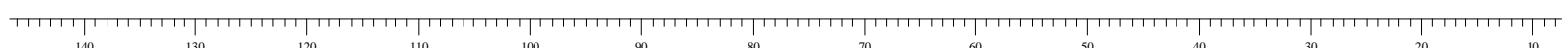



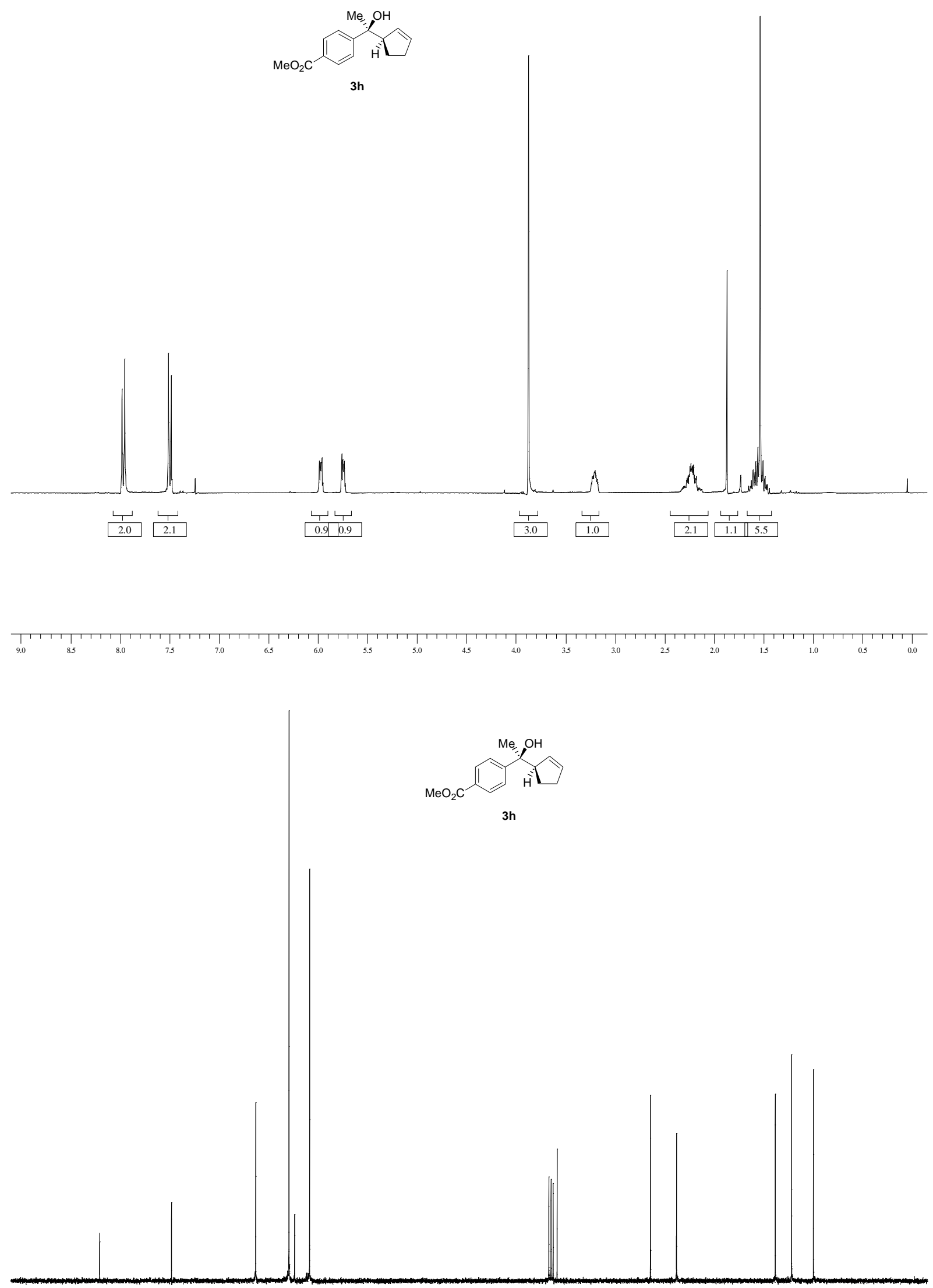

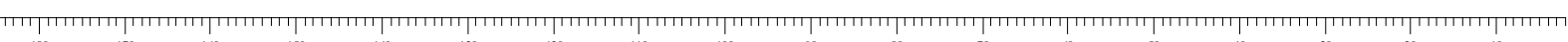

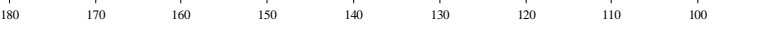


$1 \mathrm{MeO}^{\mathrm{O}}$

3i
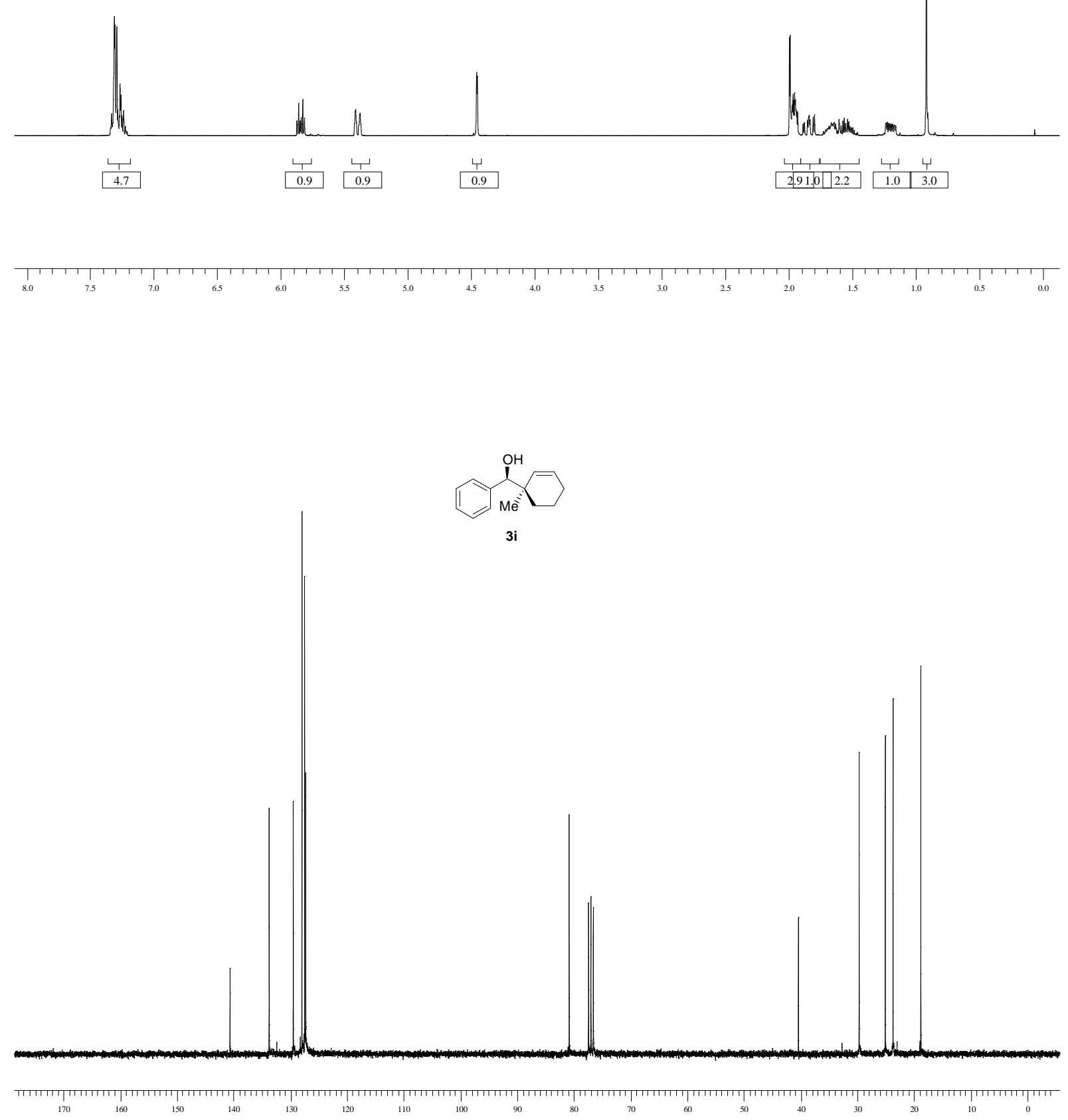


$$
\text { 3j }
$$

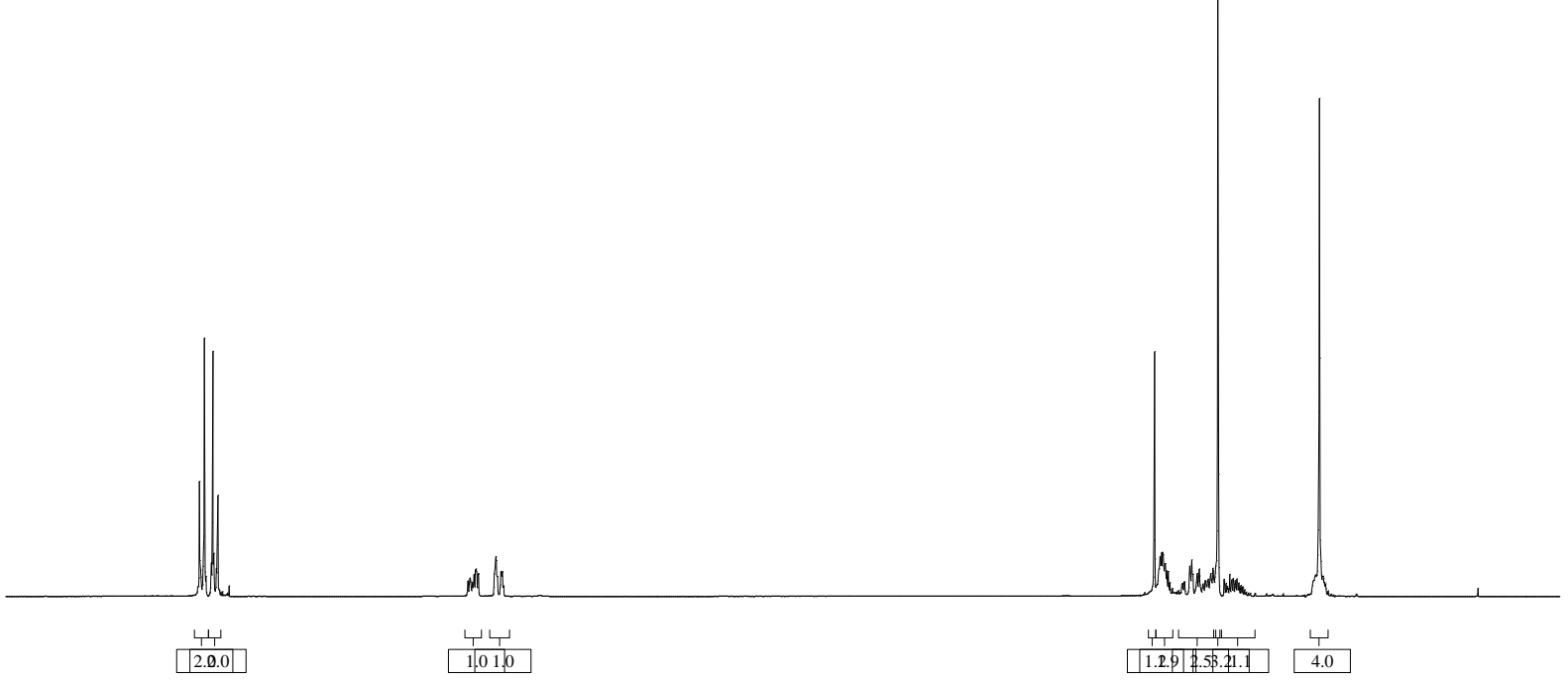

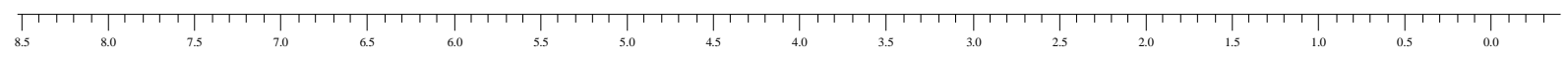<smiles>[M]C(O)(O)C(C)(C)c1ccc(Br)cc1</smiles>

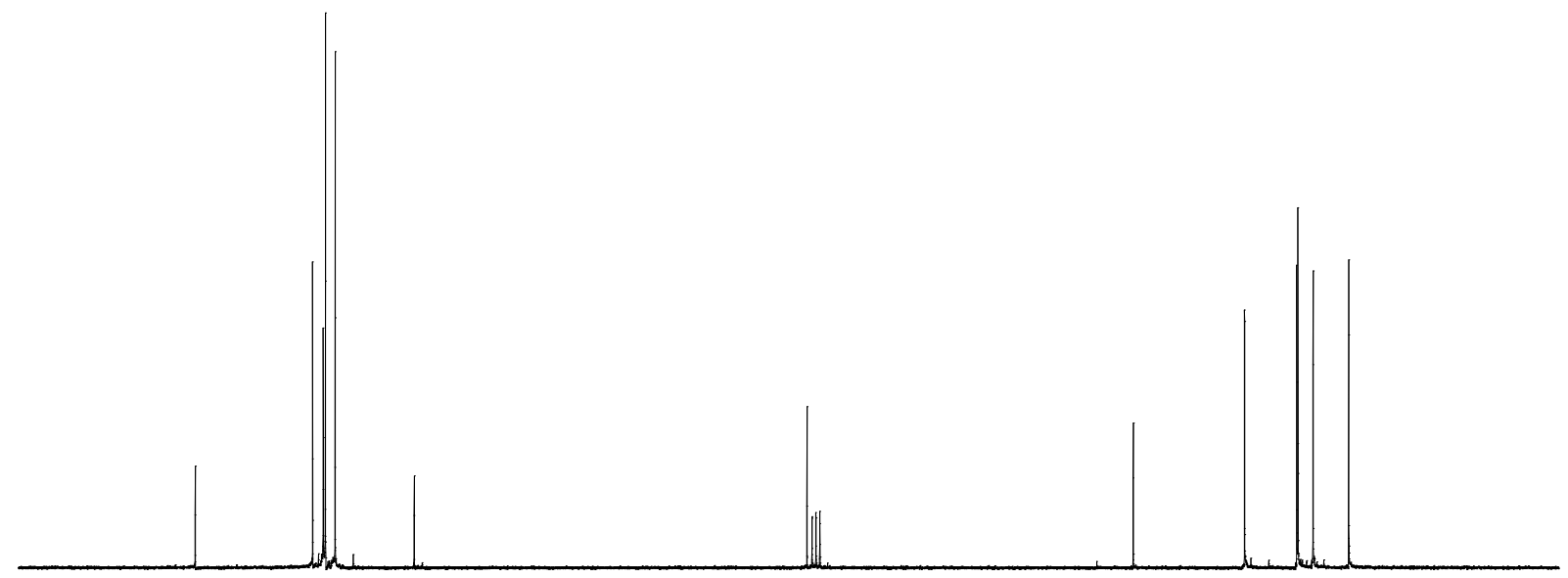



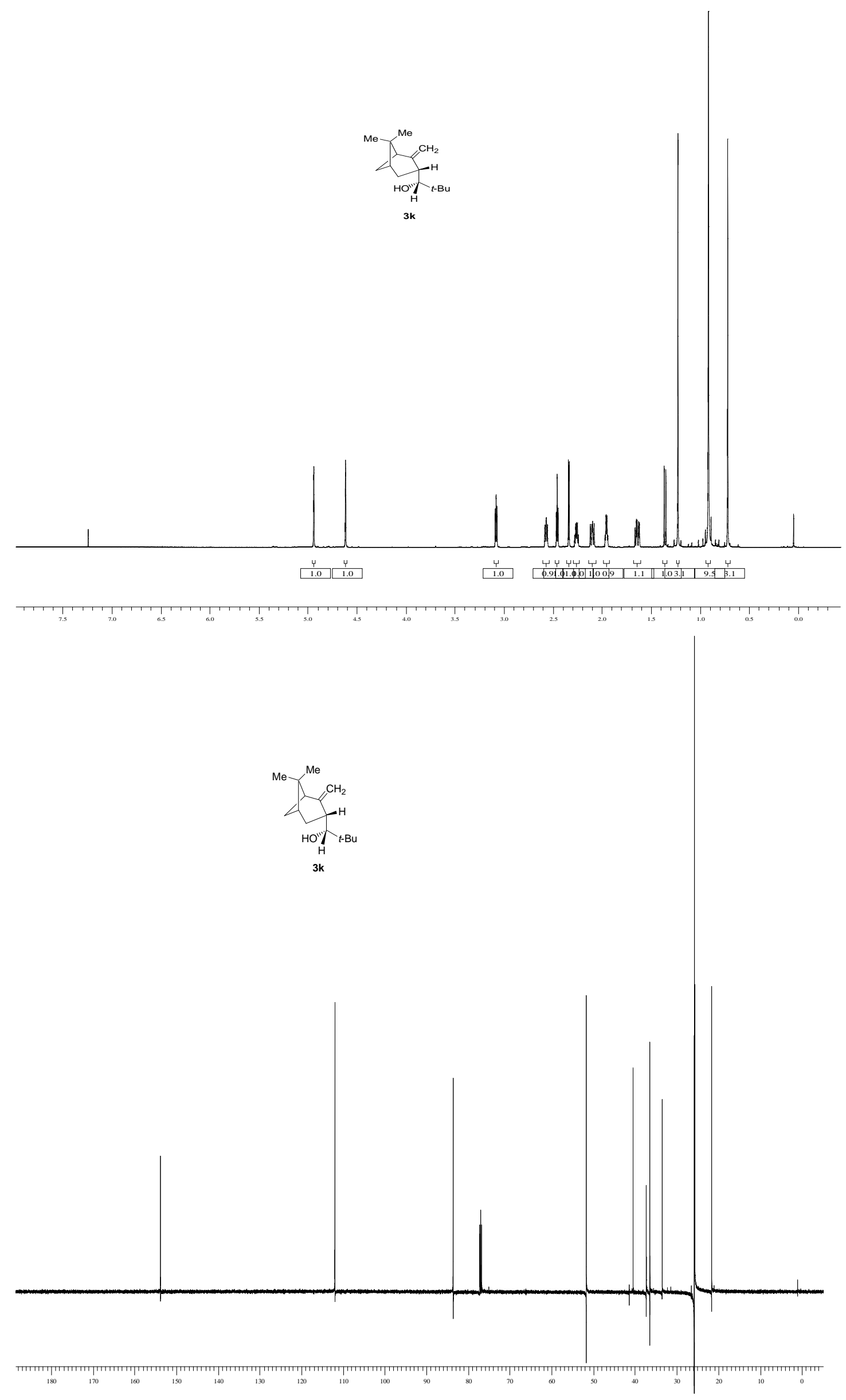

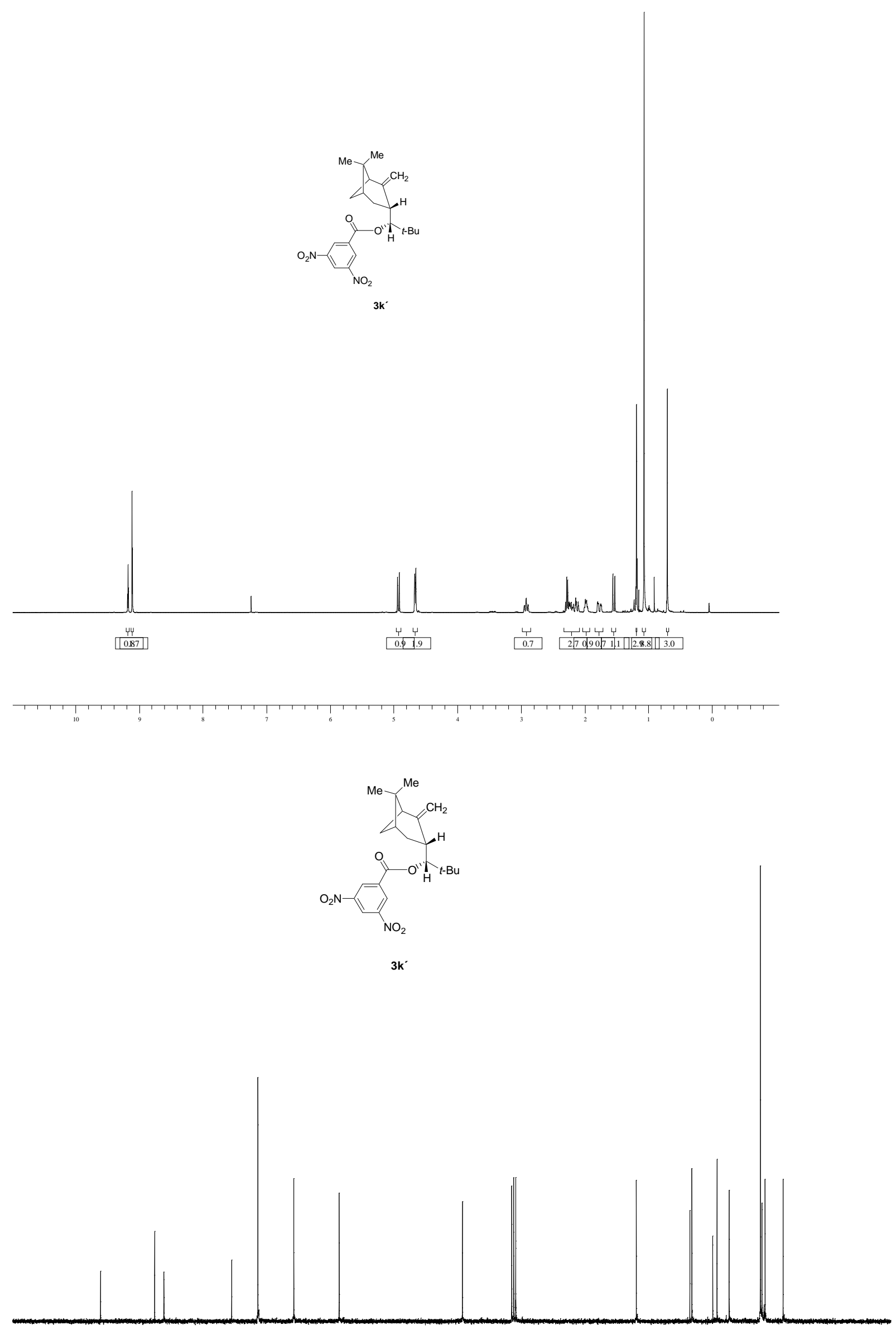

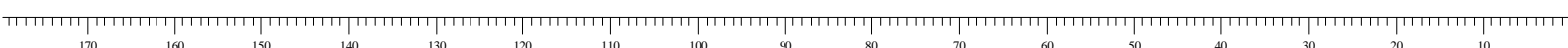

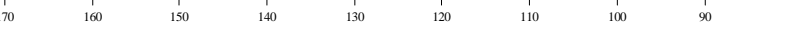



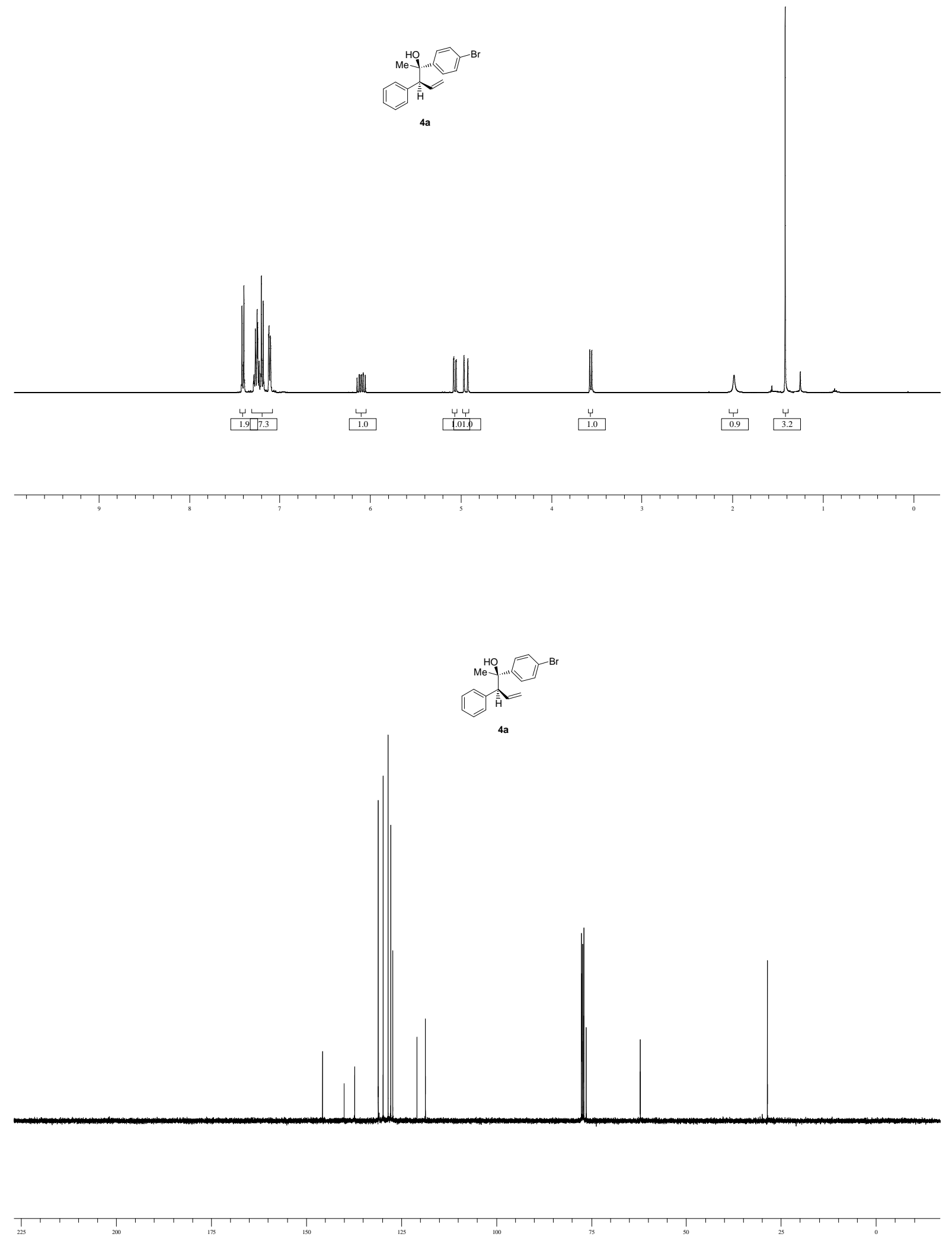

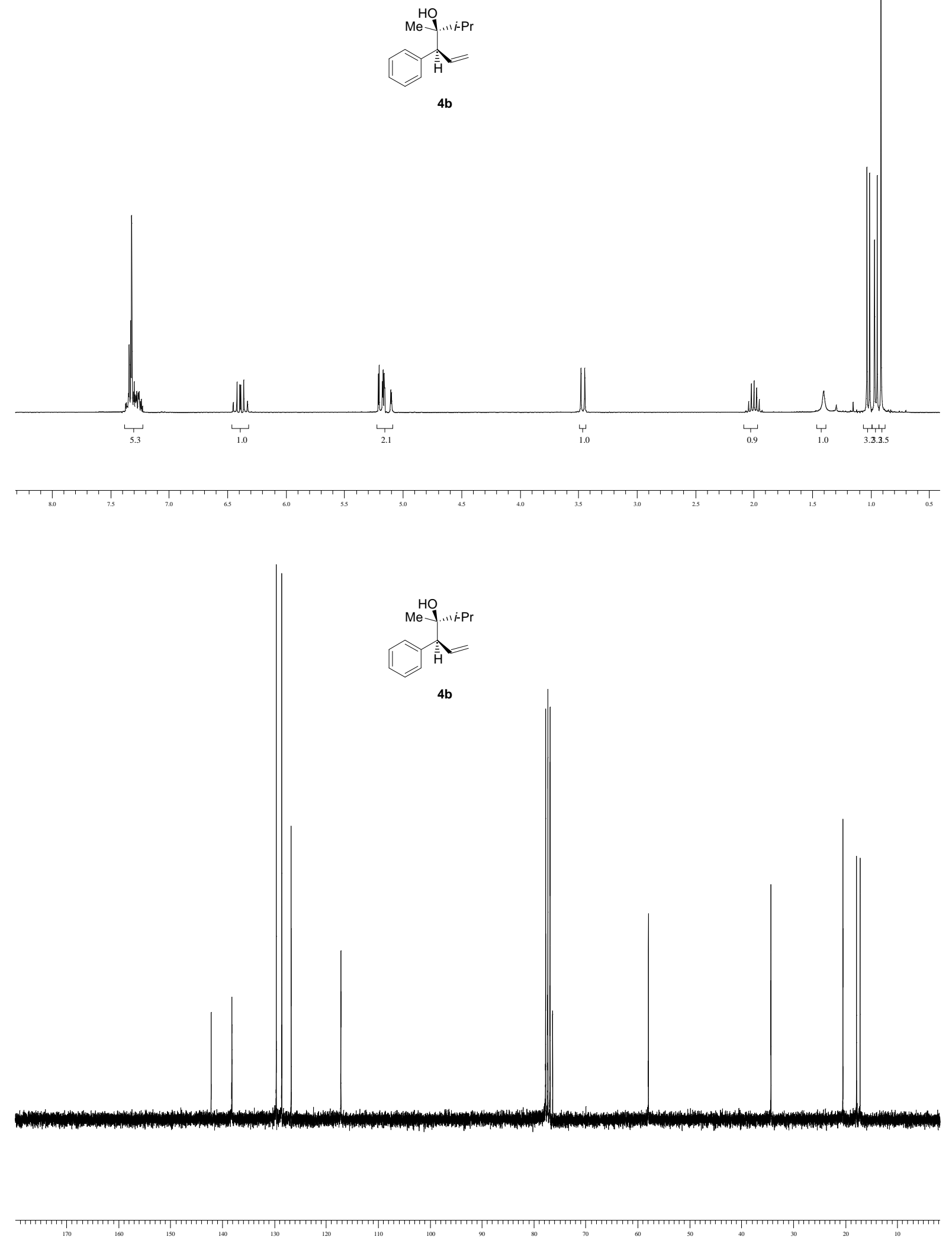

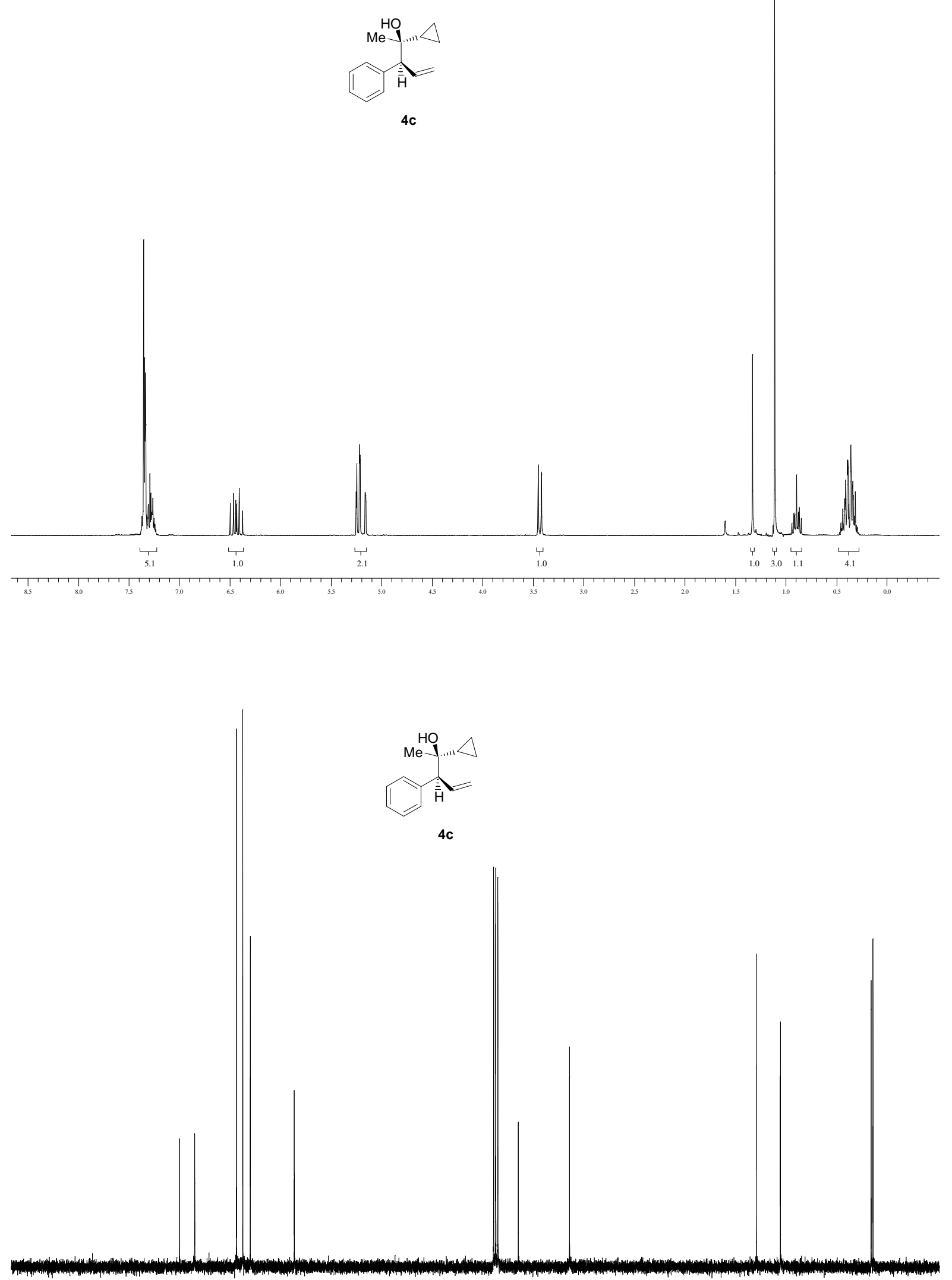

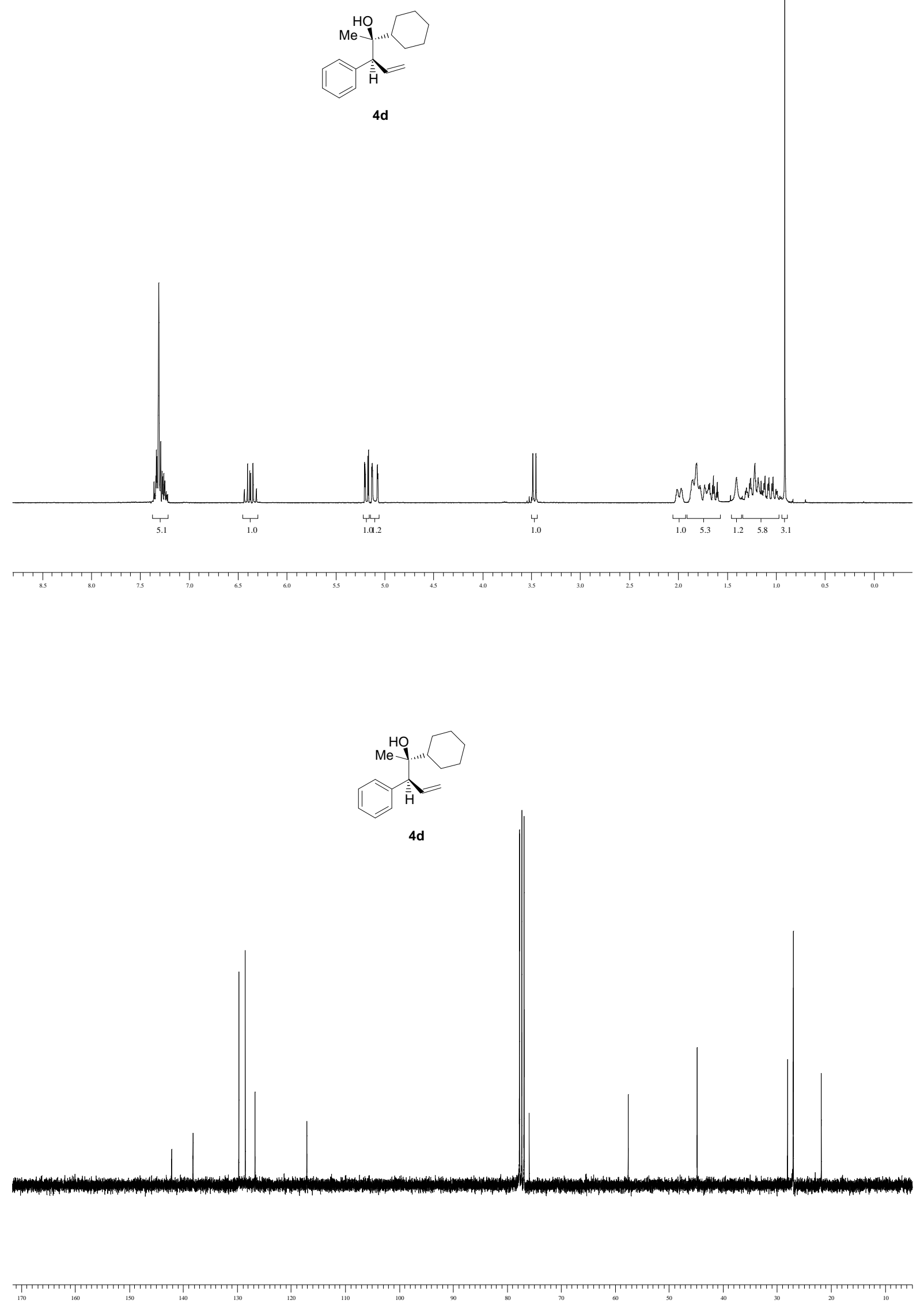

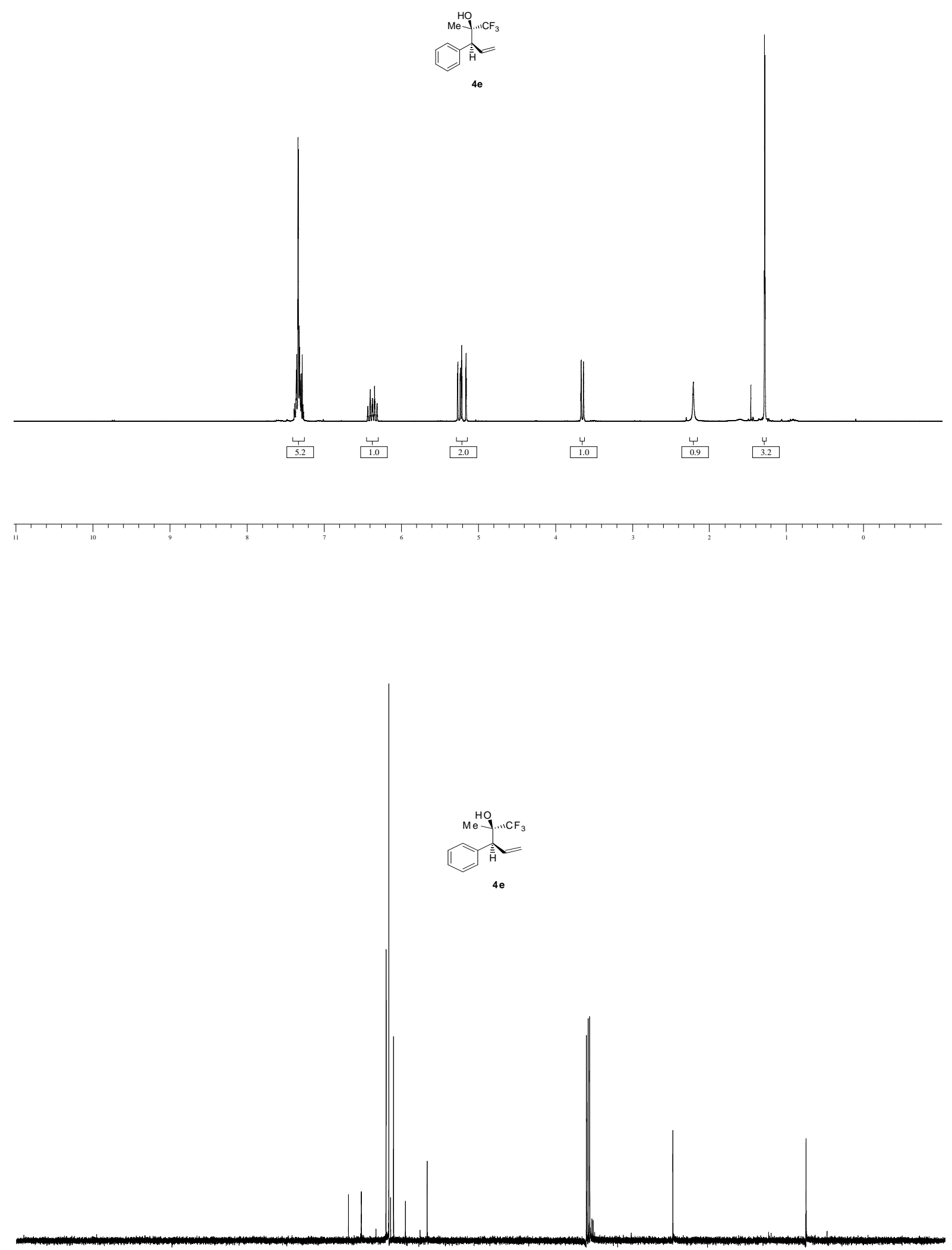

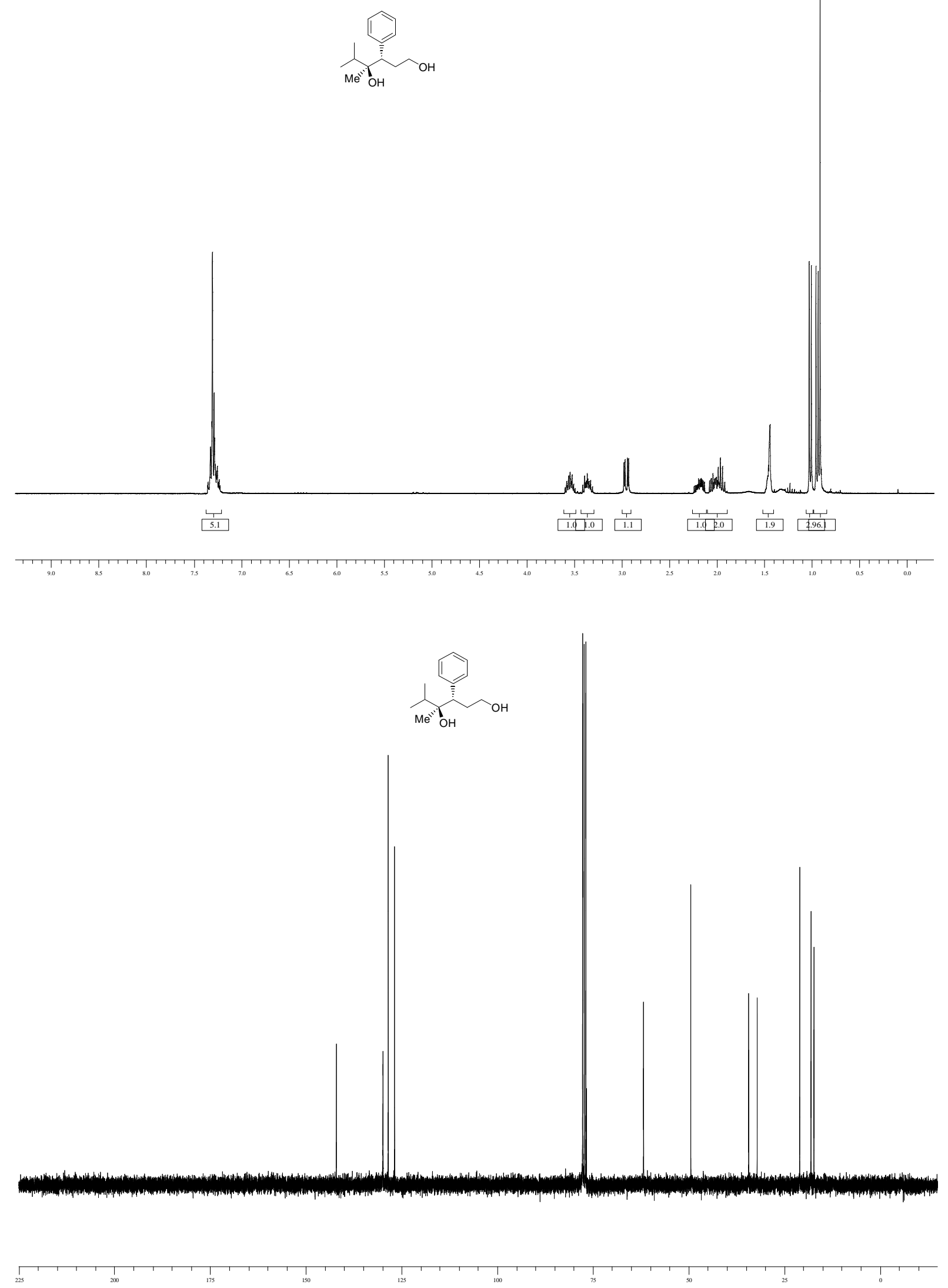

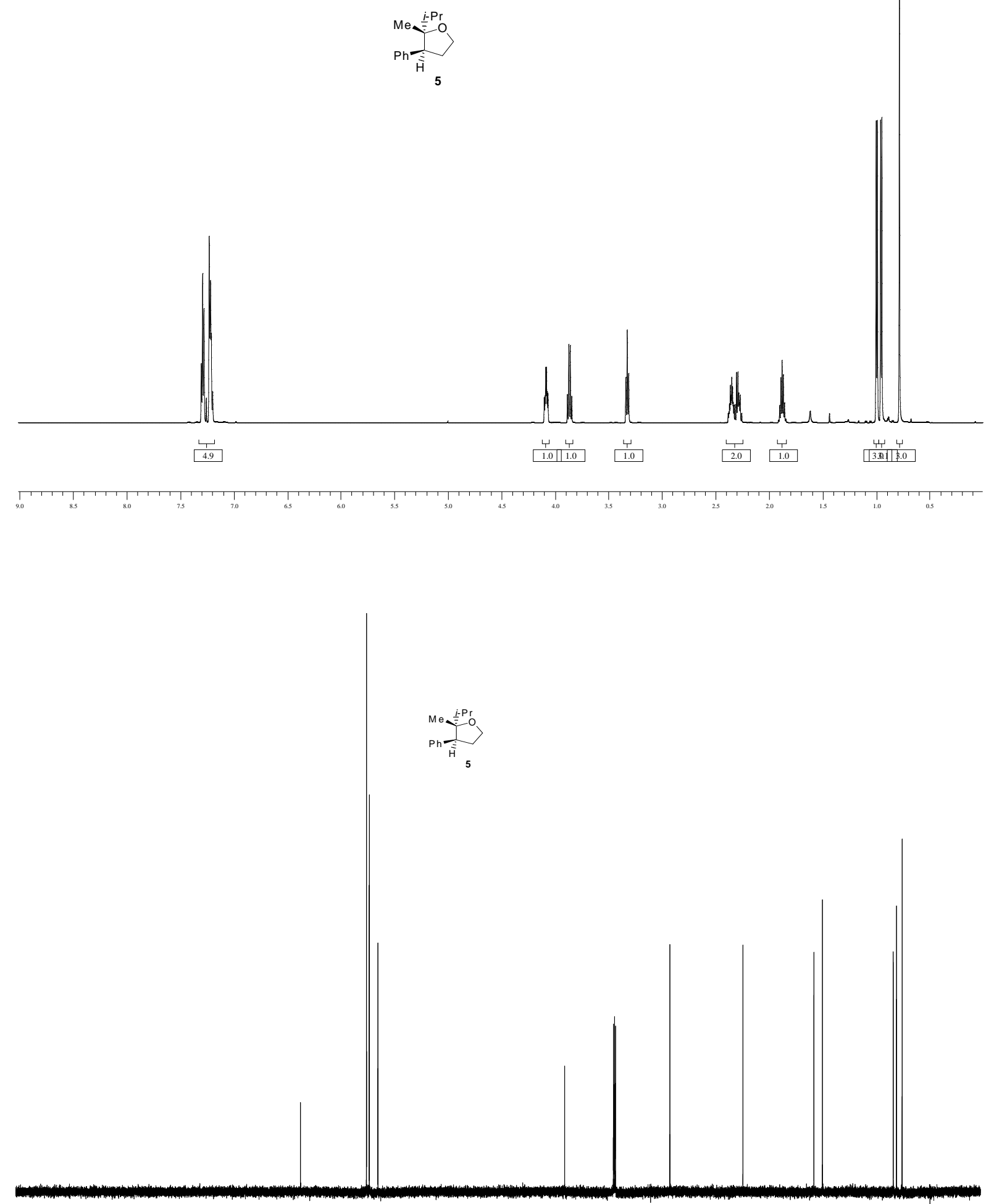

${ }_{200}^{1}$ 

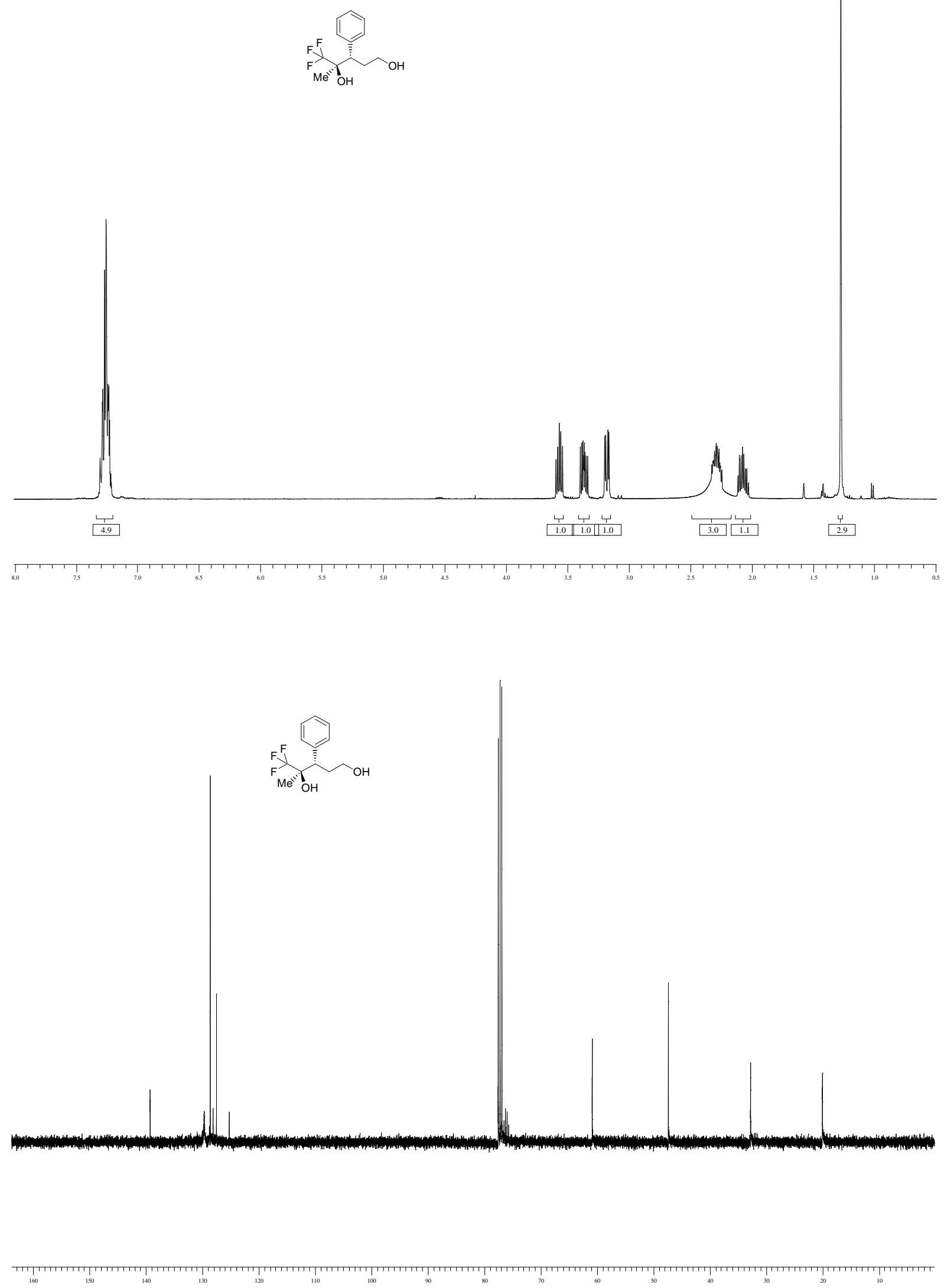

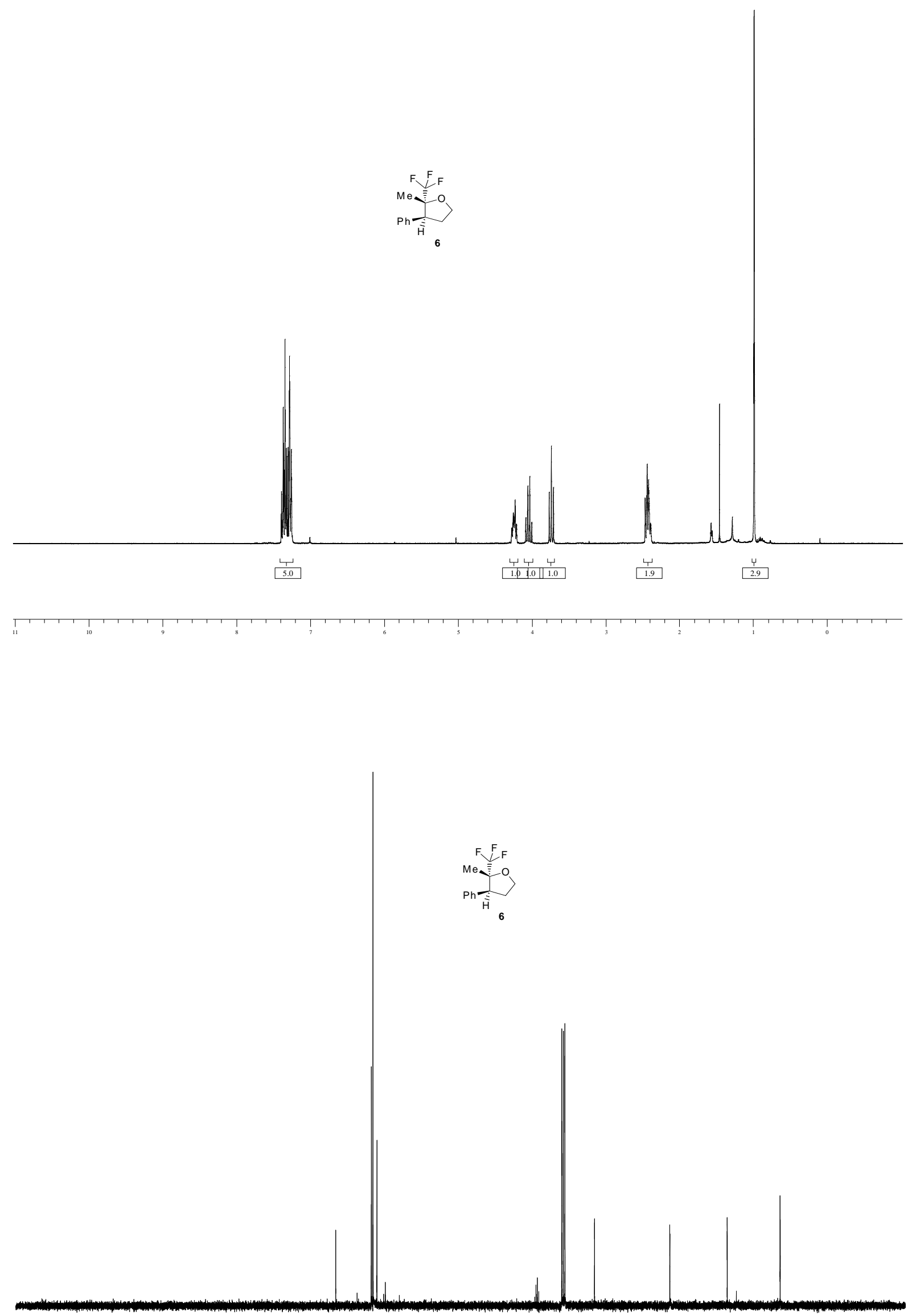

$\prod_{225}$

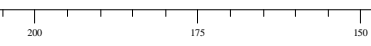

${ }_{100}^{1}$ 

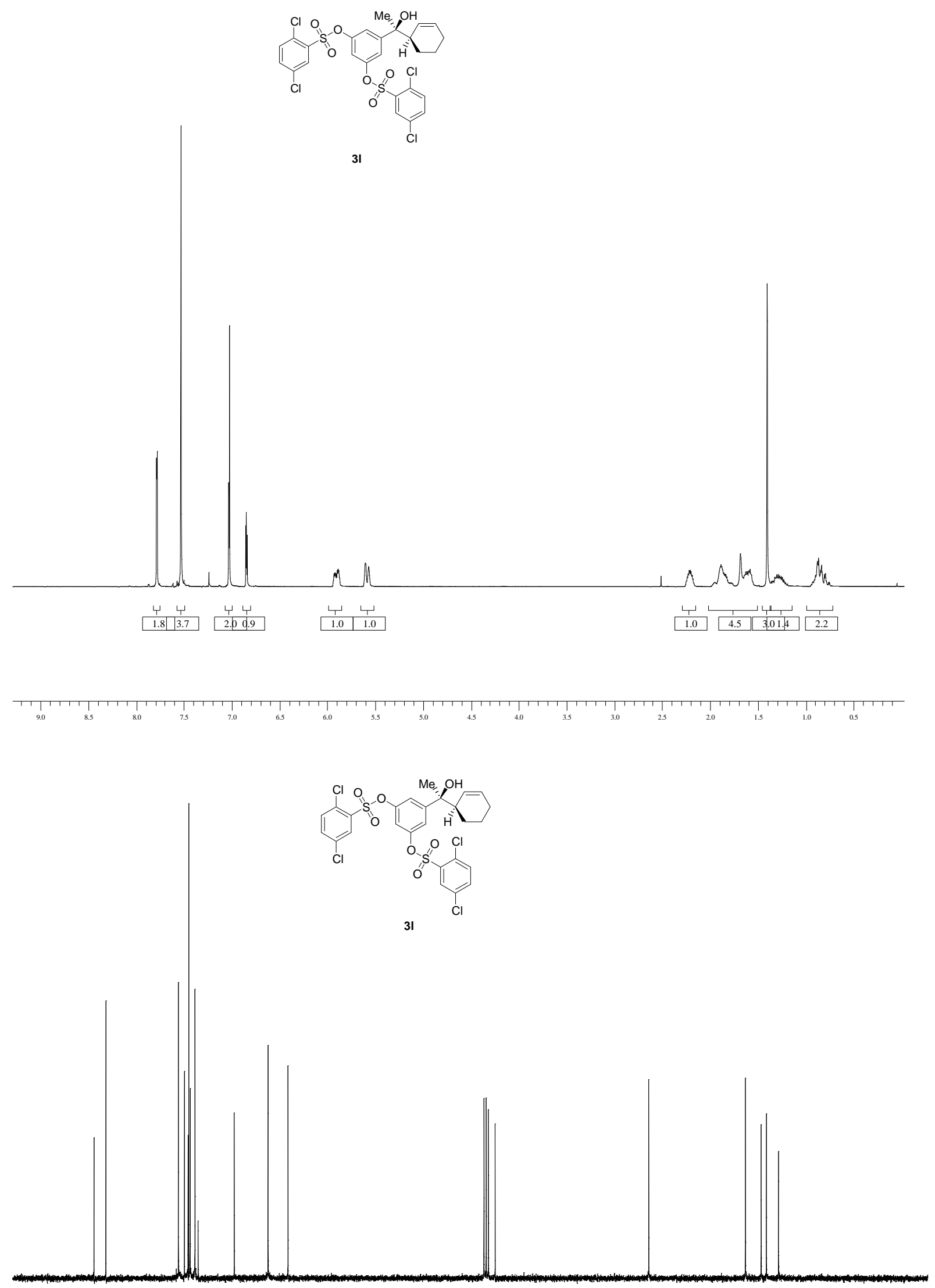